\title{
Ursachen des Hausärztemangels: ein Vergleich zwischen Frankreich und der Schweiz
}

Cerny, Thomas ; Rosemann, Thomas ; Tandjung, Ryan ; Chmiel, Corinne

\begin{abstract}
BACKGROUND AND OBJECTIVES Both France and Switzerland face a general practitioner (GP) shortage. What differences or parallels exist between the two countries with regard to the causes for this shortage? What conclusions might be drawn from a systematic comparison? METHODS Literature review with qualitative and semi-quantitative content analysis. RESULTS Parallels exist in the comparing categories work contents, working structure, income and social status, medical school formation, private life, psychological motives. Differences are found in the categories biography and social selection, medical socialisation, residency. In Switzerland, residency is not uniformly structured, rarely institutionally organised and contains only few elements specific to general medicine. In France, medical socialisation not only exalts the specialists, but also strongly devaluates the GPs. CONCLUSIONS By systematic analysis and comparison of both countries' pertinent literature, France and Switzerland can deepen their understanding of GP shortage. This paper identifies possible fields of action from medical school through residency up to workplace conditions that are pivotal in addressing the shortage of GPs.
\end{abstract}

DOI: https://doi.org/10.1024/1661-8157/a002362

Other titles: Reasons for General Practitioner Shortage - a Comparison Between France and Switzerland

Posted at the Zurich Open Repository and Archive, University of Zurich

ZORA URL: https://doi.org/10.5167/uzh-127252

Journal Article

Accepted Version

Originally published at:

Cerny, Thomas; Rosemann, Thomas; Tandjung, Ryan; Chmiel, Corinne (2016). Ursachen des Hausärztemangels: ein Vergleich zwischen Frankreich und der Schweiz. Praxis, 105(11):619-636.

DOI: https://doi.org/10.1024/1661-8157/a002362 


\section{Praxis}

\section{Ursachen des Hausärztemangels - Ein Vergleich zwischen Frankreich und der Schweiz \\ --Manuskript-Entwurf--}

\begin{tabular}{|c|c|}
\hline Manuskriptnummer: & PRAXIS-D-15-00134R1 \\
\hline Schlüsselwörter: & $\begin{array}{l}\text { Hausärztemangel } \\
\text { Allgemeinmedizin } \\
\text { Frankreich } \\
\text { Schweiz } \\
\text { Systematischer Vergleich }\end{array}$ \\
\hline Korrespond. Autor: & $\begin{array}{l}\text { Corinne Chmiel } \\
\text { Institut für Hausarztmedizin } \\
\text { SWITZERLAND }\end{array}$ \\
\hline \multicolumn{2}{|l|}{$\begin{array}{l}\text { Korrespondierender Autor, zweite } \\
\text { Institution: }\end{array}$} \\
\hline Erstautor: & Thomas Cerny \\
\hline \multicolumn{2}{|l|}{ Erstautor, Zweitinformationen: } \\
\hline \multirow[t]{3}{*}{ Reihenfolge der Autoren: } & Thomas Cerny \\
\hline & Thomas Rosemann \\
\hline & Ryan Tandjung \\
\hline Antwort an Gutachter: & $\begin{array}{l}\text { Sehr geehrter Gutachter } \\
\text { Im Namen der Co-Autoren bedanke ich mich herzlichst dafür, dass Sie unsere Arbeit } \\
\text { als interessant, sehr wertvoll und gut recherchiert beurteilen. Ihre inhaltlichen Inputs } \\
\text { haben wir versucht bestmöglichst umzusetzten: Tabelle } 2 \text { wurde in die Anhäge } \\
\text { "verbannt" und die vorgeschlagenen Kürzungen wurden vorgenommen. Die Details } \\
\text { sind im angehänten revidierten Manuskript inklusive Track Changes ersichtlich. Die } \\
\text { darstellerischen Probleme (Auflösung) werden wir im Rahmen des Type-Setting }\end{array}$ \\
\hline
\end{tabular}


Prozesses angehen und sollten meines Erachtens keine grösseren Probleme darstellen.

Vielen Dank für Ihre Bereitschaft unser Manuskript zu revidieren.

Freundliche Grüsse

PD Dr. med. Corinne Chmiel 


\section{Key Messages}

Diese Arbeit zeigt konkrete Massnahmen auf, wie dem HA-Mangel aktiv entgegengewirkt werden kann:

- Veränderung der medizinischen Sozialisierung zu Gunsten der HA-Medizin

- Mehr Präsenz der HA-Medizin-spezifischen Inhalte im Studium

- national einheitlich strukturierte und organisierte Weiterbildung

- Genügend lange Weiterbildungszeit

- Förderung der Anpassung der Arbeitsstrukturen an veränderte Bedürfnisse (bei gleichzeitiger Wahrung der beruflichen Autonomie)

- Angleichung der Generalisten- an die Spezialistengehälter 


\section{Englische Zusammenfassung}

Background and objectives: Both France and Switzerland face a general practitioner (GP) shortage. What differences or parallels exist between the two countries with regard to the causes for this shortage? What conclusions might be drawn from a systematic comparison? Methods: Literature review with qualitative and semi-quantitative content analysis.

Results: Parallels exist in the comparing categories work contents, working structure, income and social status, medical school formation, private life, psychological motives. Differences are found in the categories biography and social selection, medical socialisation, residency. In Switzerland, residency is not uniformly structured, rarely institutionally organised and contains only few elements specific to general medicine. In France, medical socialisation not only exalts the specialists, but also strongly devaluates the GPs.

Conclusions: By systematic analysis and comparison of both countries' pertinent literature, France and Switzerland can deepen their understanding of GP shortage. This paper identifies possible fields of action from medical school, through residency, up to workplace conditions that are pivotal in addressing the shortage of GPs.

\section{Key Words}

General practitioner shortage

Family Medicine

France

Switzerland

Systematic comparison 


\section{Résumé}

Introduction: La Suisse aussi bien que la France sont confrontés à une pénurie de médecins généralistes. Y a-t-il des différences/parallèles entre les deux pays concernant les déterminants? Que peut-on y apprendre par une comparaison systématique?

Méthodes: Revue de littérature et exploitation qualitative et sémi-quantitative selon la méthode d'analyse du contenu.

Résultats: Des parallèles existent entre les catégories «contenus du travail», «conditions de travail», «facteurs économiques», «statut social», «formation prégraduée universitaire», «vie privée» et «motifs psychiques». Des différences subsistent entre les catégories «biographie», «sélection sociale», «Socialisation médicale» et «formation postgraduée». En Suisse, cette dernière n'est pas unitairement structurée, a peine institutionnalisée et peu spécifique pour la médicine générale. En France, la socialisation médicale non seulement survalorise les spécialistes, mais aussi déprécie fortement les médecins généralistes. Conclusion: La comparaison systématique de la littérature pertinente permet aux deux pays une apprentissage mutuelle révélatrice. Ce travail formule des mesures concrètes - de la formation universitaire jusqu'à l'organisation du cabinet en passant par la formation postgraduée - pour faire face à la manque de médecins généralistes.

\section{Mots-clés:}

médecine de famille

pénurie de médecins généralistes

France

Suisse

Comparaison systématique 
Institut für Hausarztmedizin, Universitätsspital Zürich

T. Cerny, T. Rosemann, R. Tandjung, C. Chmiel

Ursachen des Hausärztemangels - Ein Vergleich zwischen Frankreich und der Schweiz

Reasons for General Practitioner Shortage - A Comparison between France and Switzerland

Im Artikel verwendete Abkürzungen:

\begin{tabular}{|l|l|}
\hline ECN & épreuves classantes nationales \\
\hline EHCI & Euro Health Consumer Index \\
\hline HA & Hausarzt, Hausärzte \\
\hline MEP & mode d'exercise particulier \\
\hline
\end{tabular}




\section{Ü1: Zusammenfassung}

Hintergrund und Fragestellung: Sowohl Frankreich als auch die Schweiz beklagen einen Hausärzte(HA)mangel. Bestehen Unterschiede/Parallelen zwischen den Ursachen in beiden Ländern? Was können wir aus einem systematischen Vergleich lernen? Methodik: Literaturreview mit qualitativer und semiquantitativer Inhaltsanalyse. Ergebnisse: Parallelen bestehen in den Kategorien Arbeitsinhalte, Arbeitsstrukturen, Ökonomie, Sozialstatus, Ausbildung, Privatleben, psychische Motive. Unterschiede bestehen in den Kategorien Biografie, soziale Selektion, medizinische Sozialisation, Weiterbildung. Letztere ist in der Schweiz nicht einheitlich strukturiert, selten institutionell organisiert, enthält wenig HA-Medizin-spezifische Inhalte. Die medizinische Sozialisierung in Frankreich überhöht im Unterschied zur Schweiz nicht nur die Spezialisten, sondern entwertet stark die HA-Medizin.

Schlussfolgerungen: Durch die systematische Literatur-Analyse können beide Länder mehrfach voneinander lernen. Diese Arbeit zeigt konkrete Massnahmen vom Studium über Weiterbildung bis zur hausärztlichen Arbeitsstruktur auf, wie dem HA-Mangel aktiv entgegengewirkt werden kann.

Background and objectives: Both France and Switzerland face a general practitioner (GP) shortage. What differences or parallels exist between the two countries with regard to the causes for this shortage? What conclusions might be drawn from a systematic comparison? Methods: Literature review with qualitative and semi-quantitative content analysis.

Results: Parallels exist in the comparing categories work contents, working structure, income and social status, medical school formation, private life, psychological motives. Differences are found in the categories biography and social selection, medical socialisation, residency. In Switzerland, residency is not uniformly structured, rarely institutionally organised and contains only few elements specific to general medicine. In France, medical socialisation not only exalts the specialists, but also strongly devaluates the GPs.

Conclusions: By systematic analysis and comparison of both countries' pertinent literature, France and Switzerland can deepen their understanding of GP shortage. This paper identifies possible fields of action from medical school, through residency, up to workplace conditions that are pivotal in addressing the shortage of GPs. 


\section{Ü1: Einleitung}

Längst ist auch in der Schweiz der Hausärzte(HA)-Mangel für viele zu einer unangenehmen Perspektive geworden. Für die Bevölkerung, weil sie um die Konstanz und

Personenbezogenheit ihrer künftigen medizinischen Betreuung besorgt ist; für Gesundheitsökonomen, weil eine flächendeckende Primärversorgung erwiesenermassen die Gesundheitskosten senkt und gleichzeitig die Qualität steigert [1-4]; für die Medizin selbst, weil mit dem Verschwinden der HA-Medizin eine der letzten Generalisten-Disziplinen verschwinden würde. Wie real diese Perspektive ist, zeigen die jüngsten Zahlen: $48 \%$ der aktuellen HA werden in zehn Jahren pensioniert [5]. Um den Status quo an HA zu halten, müssten pro Jahr 300 neue HA ihre Tätigkeit aufnehmen; 2010 waren es lediglich 88 [6]. Auf 2030 hochgerechnet resultiert in Konsultationen/Jahr ausgedrückt eine Abnahme des Angebots von 21 auf 13 Mio. bei einer Zunahme der Nachfrage von 21 auf 23 Mio [7]. Um wirksam Gegensteuer geben zu können, müssten die Ursachen des HA-Mangels zunächst möglichst vollständig verstanden sein. Hierfür bietet sich unser Nachbarland Frankreich an, da sich die Gesundheitswesen beider Länder grundsätzlich relativ ähnlich sehen und auch in Frankreich ein HA-Mangel bevorsteht. Die Fragestellung dabei lautet: Gibt es Unterschiede respektive Parallelen zwischen den Ursachen des HA-Mangels in Frankreich und in der Schweiz? Lassen sich aus einem systematischen Vergleich ein besseres generelles Verständnis der Ursachen des HA-Mangels gewinnen und allenfalls konkrete Massnahmen daraus ableiten?

\section{Ü1: Methodik}

Zur Beantwortung dieser Fragestellung erfolgte im Sinne einer qualitativen Inhaltsanalyse [8, 9] ein systematischer Vergleich der einschlägigen Publikationen zur Situation in beiden Ländern. Die Literaturauswahl erfolgte wie folgt: französische und Schweizer quantitative oder qualitative Originalarbeiten oder Mini-Reviews, die die Ursachen des HA-Mangels untersuchen, Publikationszeitpunkt ab 2004. Im Jahr 2004 fand in Frankreich eine grosse Strukturreform der ärztlichen Ausbildung statt, welche auch für die Berufswahl hin zum HA neue Realitäten schuf. Ältere Arbeiten sind somit inhaltlich überholt. Die Literatursuche fand statt in PubMed, Google Scholar, in den Suchmaschinen der einschlägigen Journals des jeweiligen Landes und schliesslich in den Literaturverzeichnissen der als Treffer gewerteten Arbeiten. Bei der Inhaltsanalyse wurde einerseits eine quantitative Strategie verfolgt (wie oft wird eine Kategorie genannt?), andererseits eine qualitative (welche konkreten Inhalte finden 
sich innerhalb einer Kategorie nach erfolgter Zuteilung?). Die zwei resultierenden Aufstellungen ermöglichten schliesslich den systematischen Vergleich.

\section{Ü1: Setting}

Frankreich und die Schweiz haben in den Grundzügen ähnliche Gesundheitswesen betreffend allgemeiner Struktur, ärztlicher Versorgungssituation, HA-Mangel, Ausbildung der Ärzteschaft, Kosten und Finanzierung. Eine Übersicht sowie weitere Eckdaten liefert Tabelle 1. Grundkenntnisse des Schweizer Gesundheitswesens werden vorausgesetzt, weswegen hier das Augenmerk auf schweizerische Besonderheiten und auf das weniger bekannte französische System gelegt wird.

\section{Ü2: Schweiz [10-12][10-12]}

In den letzten Jahren findet zunehmend eine zweifache Umverteilung von Ärzten statt: einerseits eine solche von ambulant nach stationär (+15\% seit 2003), andererseits eine Umverteilung innerhalb des ambulanten Sektors in Form einer Zunahme von Spezialisten bei Abnahme von Grundversorgern. Diese doppelte Umverteilung schlägt sich in einer Überalterung der Grundversorger nieder. Regionale Unterschiede in der Ärztedichte bestehen einerseits kantonal, andererseits im Vergleich urban versus ländlich. Dies lässt sich teilweise mit der fortschreitenden Feminisierung der Ärzteschaft begründen: Der Frauenanteil in der berufstätigen Ärzteschaft stieg in der letzten Dekade von 29\% (2000) auf 37,5\% (2012), unter den Assistenzärzten machte der Frauenanteil im Jahr 2010 bereits 56\% aus. Dabei wird nun beobachtet, dass Ärztinnen bevorzugt in urbanen Gebieten tätig sind (mehr Möglichkeiten der Teilzeitarbeit, bessere Kinderbetreuung) [13, S. 25]. Ärztedemographisch auffällig ist weiter der hohe Ausländeranteil in der Schweizer Ärzteschaft: $40 \%$ im stationären, $17 \%$ im ambulanten Sektor.

Zusammenfassend lässt sich sagen, dass nicht zu Letzt dank hohem Ausländeranteil der allgemeine Ärztebedarf gedeckt ist [14], jedoch zunehmend ein Fachrichtung-bezogenes und örtliches Verteilungsproblem vorliegt. Dies gilt besonders für den bevorstehenden HAMangel. Die aktuelle Verteilung der HA weist eine überdurchschnittliche HA-Dichte in urbanen Gebieten auf, hingegen eine unterdurchschnittliche Dichte in peripheren Gebieten. Bezieht man jedoch die Arbeitspensen mit ein, ergibt sich ein nivelliertes Bild: in urbanen Zentren wird die hohe Dichte von mehr Teilzeitarbeit begleitet, wodurch die verfügbare ärztliche Versorgung auf das Landesmittel sinkt. In den ländlichen Regionen wird die niedrige HA-Dichte von hohen Arbeitspensen begleitet, dank derer das Landesmittel der ärztlichen Versorgung erreicht wird. Allerdings verfügen die ländlichen Regionen somit über 
eine geringe „Elastizität des Angebots“ [13, S.24], da im Falle einer steigenden Nachfrage oder einer Praxisschliessung die bereits ausgelasteten HA die Lücke nicht kompensieren können. Beide Szenarien werden gemäss Prognosen jedoch eintreten [7, S.70-81]: während die Anzahl HA schweizweit von 6'500 (2010) auf 5'700 (2030) abnimmt, nimmt die Nachfrage nach Konsultationen bis 2030 um 10\% zu.

Details zum Medizinstudium und zur Weiterbildung können Tabelle 1 entnommen werden. Die Anzahl Studienanfänger stagniert seit 1980 um 1'000 pro Jahrgang, seit 1998 werden die Studienplätze durch einen Numerus clausus knapp unter der 1000er Marke gehalten, während die Nachfrage nach Plätzen seither steigt [15].

Details zu den Kosten, zur Finanzierung und zur Qualität des Schweizer Gesundheitswesens können ebenfalls Tabelle 1 entnommen werden.

\section{Ü2: Frankreich [16-18]}

Auch das französische Gesundheitswesen basiert auf dem Sozialversicherungsmodell. Es folgt dem sozialen Gedanken stärker, indem die obligatorischen Versicherungsbeiträge einkommensabhängig sind. Gleichzeitig finden sich Elemente eines nationalen Gesundheitsdienstes (sog. „Beveridge“-System, wie z.B. in England), indem die Bedeutung der Finanzierung via Steuern zunimmt und der universelle Zugang zur Gesundheitsversorgung in grösserem Ausmass garantiert wird: 2008 waren 99,9\% der Bevölkerung durch die gesetzliche Krankenkasse gedeckt (darin eingeschlossen Fremde de passage und illegale Einwanderer.)

Auch in Frankreich zeichnet sich in den letzten Jahren die doppelte Umverteilung ab: zum einen eine stärkere Zunahme von Spezialisten $(+14,8 \%)$ als von Generalisten $(+6,9 \%)$, zum anderen eine Umverteilung von ambulant nach stationär [19, S. 15-17]. So haben z.B. die Bestände der niedergelassenen Generalisten nur um 0,1\% zugenommen, gegenüber einem Plus von $29 \%$ bei den in Krankenhäusern angestellten Generalisten. Ebenso beklagt auch Frankreich ein örtliches Verteilungsproblem der Ärzte: einerseits auf Ebene der régions, andererseits auf Ebene der départements. Während sich die Verteilung zwischen den Regionen stark verbessert hat, blieben zwischen den Departementen massive Dichteunterschiede bestehen: man spricht in Frankreich von sogenannten „medizinischen Wüsten" - les deserts médicaux. Ärztedemographisch betrachtet sieht man auch in Frankreich eine fortschreitende Feminisierung des Berufes: der Frauenanteil stieg von $30 \%$ (1990) auf 41\% (2012); unter den Assistenzärzten machten 2010 die Frauen bereits 60\% aus. Das allgemeine Durchschnittsalter der berufstätigen Ärzteschaft stieg von 40 J. (1990) auf $52 \mathrm{~J}$. (2012). Dieser starke Anstieg wiederspiegelt die rasante Zunahme der Ärztezahl in den 70er und 80er Jahren: jene zahlenstarken Generationen wandern in der Alterspyramide nun nach oben. Ausländische Ärzte machen nur $8,6 \%$ der französischen Ärzteschaft aus. 
Zusammenfassend lässt sich für Frankreich sagen, dass die allgemeine Ärztepopulation gegenwärtig genügend gross ist, jedoch schon seit längerem das Problem einer zunehmenden medizinischen Desertifikation besteht.

Dabei sind die HA noch am besten nach der Bevölkerungsdichte verteilt, im Gegensatz zu den anderen Grundversorgern (Gynäkologen und Pädiater) oder den Spezialisten. Doch scheint die bisher relativ gleichmässige HA-Verteilung durch die neuen Niederlassungstrends der jungen $\mathrm{HA}$ (<40 J.) künftig gefährdet: Sie konzentrieren sich in den grossen Agglomerationen [20, S.37-47]. Dabei finden sich neu universitäre Zentren, andere junge HA (als Hinweis auf Gruppenpraxen) und Kindergärten (als Hinweis auf zunehmende Bedeutung des Familienlebens) in ihrer nächsten Umgebung [20, S.49-52]. Diese Tendenz weist auf einen zukünftigen regionalen HA-Mangel hin. Aber auch national steuert Frankreich auf einen HA-Mangel zu: in der Periode 1995-2005 stagnierte die Anzahl niedergelassener HA und für die kommenden Jahre werden für 78 der 96 Festland-Departemente rückläufige Zahlen vorausgesagt [21, S.155]. Ein wesentlicher struktureller Unterschied im Bereich der HA-Medizin muss an dieser Stelle erwähnt werden: Im Gegensatz zu Schweizer HA steht französischen HA in der Regel kein Basislabor und Röntgengerät in der HA-Praxis zur Verfügung; für diese Untersuchungen müssen sie ihre Patienten in ein - meist in der Nähe gelegenes - privates Labor oder Röntgeninstitut schicken. Zudem beschäftigen nicht alle HA eine Medizinische Praxisassistentin und bewältigen demnach einen Grossteil der administrativen Arbeiten eigenhändig.

Im Ausblick auf die Versorgungssituation 2030 stehen in Frankreich drei Themen im Vordergrund:

- Die bevorstehende Pensionierung zahlenstarker Jahrgänge steht bevor. Obwohl die Anzahl Studienplätze zwischen 2000-2010 wiederholt angehoben wurde, wird aufgrund der langen ärztlichen Ausbildungszeit dennoch eine „Senke“ in der Ärztedichte entstehen. Die auch für Frankreich prognostizierte wachsende medizinische Nachfrage wird diese Senke noch verstärken.

- Die doppelte Umverteilung wird sich fortsetzen: Zunahme der Ärzte im stationären Bereich bei Abnahme im ambulanten Sektor [23] und relative Zunahme der Spezialisten gegenüber den Generalisten [21, S.15].

- Die gegenwärtig schon bestehende Konzentration der Ärzte auf UniversitätsspitalStädte wird auf Kosten der ländlichen Regionen und Städte ohne Universitätsspital weiter zunehmen.

Details zur Aus- und Weiterbildung sind in Tabelle 1 ersichtlich. Die Anzahl Studienabgänger lag 2011 bei 6'976, was einer Verdopplung im Vergleich zu 2004 entspricht [24]. Die Wahl der Fachrichtung erfolgt in Frankreich seit 2004 durch die sog. épreuves classantes nationales (ECN). Hierbei absolvieren alle Studienabgänger des Landes die gleiche 
schriftliche Prüfung, nach der eine Rangliste erstellt wird. Danach wählt jeder Student in Abfolge seines Ranges das Fach und den Ort seiner Weiterbildung. So können 46\% der Absolventen unter mindestens 23 Fachrichtungen (von insgesamt 30) wählen, 42\% hingegen nur noch aus einer Auswahl von maximal 6 Fächern. In drei Disziplinen verbleiben bis am Schluss offene Stellen: HA-Medizin, Arbeitsmedizin und Public Health. Die ECN werden nicht zuletzt dafür von namhaften Persönlichkeiten kritisiert, dass sie eine starke Hierarchisierung und folglich Wertung der Disziplinen bewirken [25]. Die anschliessende Weiterbildung dauert je nach Disziplin drei, vier oder fünf Jahre, wobei einzig im Falle der HA-Medizin die Weiterbildungsdauer drei Jahre beträgt [26].

Details zu den Kosten, zur Finanzierung und zur Qualität des französischen Gesundheitswesens sind in Tabelle 1 ersichtlich.

\section{Ü1: Resultate}

\section{Ü2: Das Interaktionsmodell und seine Kategorien}

Die qualitative Inhaltsanalyse lieferte als erste Kategorisierung die Unterteilung in extrinsische und intrinsische Faktoren: Alle Ursachen des HA-Mangels lassen sich entweder äusseren, realen Missständen oder aber inneren Ansprüchen, denen die Realität nicht gerecht wird, zuschreiben. Diesen zwei Kategorien wurden alle weiteren gefundenen Kategorien zugeordnet. Abbildung 1 liefert eine Übersicht über das resultierende System von Kategorien. Der Vollständigkeit halber enthalten sind auch die Kategorien „Gesellschaft“ und "staatliche Steuerung“, vor deren Hintergrund die übrigen extrinsischen Kategorien zu sehen sind: Gesellschaft und Staat sind Akteure, die praktisch jede andere Kategorie beeinflussen können. Abbildung 1 trägt den Titel „Interaktionsmodell“, weil die eine Seite (z.B die extrinsische) nie ohne die andere (die intrinsische) auskommt, um zu einer Ursache des Mangels zu werden - jede angetroffene Realität wird erst dann zu einer negativen, wenn sie als solche durch die angehenden Ärzte bewertet wird.

\section{Ü2: Semiquantitativer Vergleich}

Unter Verwendung der Kategorien in Abbildung 1 wurde semiquantititativ untersucht, in welchem Ausmass die einzelnen Kategorien in der Literatur des jeweiligen Landes Beachtung finden. Hierfür wurden den Kategorien zugeordnete Textpassagen gemäss ihrer Länge bewertet: wenn in einer Publikation einer Kategorie ein Textparagraph gewidmet war, erhielt sie einen Punkt; entfiel ein ganzer Abschnitt mit Übertitel auf die Kategorie, erhielt sie zwei Punkte; widmete sich hingegen die Mehrheit der Arbeit einer Kategorie, wurde dies mit drei Punkten notiert. Wie intensiv eine Kategorie über alle Arbeiten hinweg in der 
gefundenen Literatur thematisiert wird, ist in Appendix 1 (für Frankreich) resp. 2 (für die Schweiz) in tabellarischer Form dargestellt. Der „Summenwert“ jeder Kategorie wurde in Form eines Balkendiagramms visualisiert. Die Balkendiagramme von Frankreich und der Schweiz wurden für eine direkte Gegenüberstellung weiterverwendet: Die Schwankung der Balken um den Mittelwert (Summe der Balkenhöhe geteilt durch Anzahl Kategorien) wurde für jedes Land als Linie abgegriffen und in einer Grafik zusammengeführt. Das Resultat zeigt Abbildung 2. Sie ermöglicht schliesslich einen semiquantitativen Vergleich der betrachteten HA-Mangel-Literatur beider Länder:

- Frankreich zeigt im Vergleich zur Schweiz eine intensivere Auseinandersetzung mit der Kategorie „Ausbildung - Medizinische Sozialisation“

- Die Schweiz zeigt im Vergleich zu Frankreich eine intensivere Auseinandersetzung mit den Kategorien „Weiterbildung“ und „Ökonomie und Sozialstatus“.

Es muss angemerkt werden, dass mehr als die Hälfte der französischen Arbeiten, die stark bei „Ausbildung - Medizinische Sozialisation“ punkteten, überwiegend dem Thema der medizinischen Sozialisation gewidmet waren [31-34] als anderen Aspekten der Ausbildung. Der Peak kommt demnach massgeblich durch die Kategorie „Medizinische Sozialisation“ zu Stande.

\section{Ü2: Qualitativer Vergleich}

Ebenfalls unter Verwendung der Kategorien in Abbildung 1 wurde untersucht, welche konkreten Inhalte in der Literatur des jeweiligen Landes unter eine bestimmte Kategorie fallen. Zwecks Übersichtlichkeit wurden hierfür die Kategorien „Arbeitsstruktur extrinsisch“ und „Arbeitsinhalte extrinsisch“ jeweils mit ihrem intrinsischen Gegenstück zusammengelegt. Nach Zuordnung der Inhalte zu den jeweiligen Kategorien wurden sinngemäss gleichartige Aussagen zu einer Grundaussage zusammengefasst unter Beibehaltung der Autorennamen. Dieses Vorgehen wurde zunächst für jedes Land getrennt durchgeführt und in tabellarischer Form festgehalten (siehe Appendix 3 und 4). Anschliessend wurden die Quintessenzen jeder Kategorie aus beiden Ländern zusammengeführt und sich gegenübergestellt (Appendix 5 Tabelle 2):

Die Ursachen des HA-Mangels in Frankreich und in der Schweiz sehen sich inhaltlich in der Mehrheit der Kategorien (8/11) ähnlich. Dies gilt für:

○ Arbeitsinhalte: In beiden Ländern zählen die Studenten das breite Krankheitsspektrum und die privilegierte Arzt-Patient-Beziehung zu den stärksten Argumenten für die HA-Medizin. Gleichzeitig schreckt das breite Spektrum einen anderen Teil der Studenten ab wegen der grossen Verantwortung und der assoziierten Unmöglichkeit, breitflächig kompetent zu sein. Ebenfalls in beiden 
Ländern findet sich die Auffassung, dass der HA nur „banale“ Krankheiten betreut und „spannende Fälle“ weiterweist.

- Arbeitsstrukturen: In beiden Ländern stellt die (interdisziplinäre) Gruppenpraxis das neue Ideal der Arbeitsstruktur dar. Sie bietet sozialen und fachlichen Austausch anstatt beruflicher Isolation, bessere Kontrolle und Flexibilität bezüglich Arbeitszeit anstatt aufopferungsvoller Pensen und schliesslich ein Teilen der ökonomischen Investitionen. In beiden Ländern wird jedoch berichtet, dass die Realität noch nicht den Vorstellungen der neuen Generation entspricht; so gelten weiterhin unkontrollierbare Arbeitszeiten und Einzelgängertum als starke Demotivatoren, deren Verbesserung hingegen als wichtigste Möglichkeit der Attraktivitätssteigerung. Dennoch wird in beiden Ländern eine Transition beobachtet: 67\% (Frankreich) respektive 70\% (Schweiz) der jungen HA arbeiten in Gruppenpraxen, mit durchschnittlich kürzeren Arbeitspensen.

○ Ökonomie und Sozialstatus: In beiden Ländern existiert eine Lohnschere zwischen niedergelassenen HA und Spezialisten, in Frankreich klafft diese noch weiter auf. Das im Vergleich geringere Einkommen wird von Studenten beider Länder zu den häufigsten Gründen gegen die Wahl der HA-Medizin gezählt. Ebenfalls in beiden Ländern werden die mit der Praxistätigkeit verbundenen Investitionen und wiederkehrenden Kosten als abschreckend bezeichnet, zumal der Lohn im Vergleich zu den Spezialisten tiefer ist. Bezüglich Sozialstatus findet sich in der Schweiz eine Arbeit, in der 30\% der befragten Medizinstudenten „fehlende soziale Anerkennung" [35] als Hindernis der HA-Medizin-Wahl angeben.

- Ausbildung: In beiden Ländern ist die HA-Medizin im Studium zeitlich und inhaltlich untervertreten. In Frankreich ist dies allerdings noch deutlich ausgeprägter, da das HA-Praktikum (=klinischer Kurs in einer HA-Praxis) im Gegensatz zur Schweiz bisher kaum etabliert war. So geben $80 \%$ der französischen Medizinstudenten an, die Realität der HA-Medizin nicht zu kennen. Aus beiden Ländern wird berichtet, dass die für die HA-Medizin wichtigen betriebswirtschaftlichen und gesundheitsökonomischen Kenntnisse im Studium zu wenig gelehrt werden.

- Privatleben: In beiden Ländern wird eine deutliche Verschiebung im Gleichgewicht Arbeit <-> Privatleben zu Gunsten der Familie beobachtet. Dies tradiert das zeitintensive, männerzentrierte Arbeitsmodell älterer HA-Generationen.

Französische Autoren beschreiben als Auslöser die Berufstätigkeit der Frau und die damit verbundene neue Rollenverteilung und Identitätsbildung des Mannes.

- Psychische Motive: In beiden Ländern zeigt sich unter den Assistenzärzten eine Angst vor definitiver Festlegung und einer damit verbundenen vorzeitigen Verwurzelung. Französische Arbeiten stellen einen Zusammenhang her mit einer 
kürzlich erhobenen „generationellen Norm der europäischen Jugend“ [36, 37] die sich durch Ausprobieren von Möglichkeiten, Aufschieben von Engagement und Vermeiden des Definitiven auszeichne.

Folgende Kategorien weisen Unterschiede zwischen Frankreich und der Schweiz auf:

- Biografie und soziale Selektion: In beiden Ländern wird das weibliche Geschlecht als Biografie-bestimmender Faktor am häufigsten thematisiert, vor allem in Zusammenhang mit dem Bedürfnis nach einem Teilzeit-Arbeitspensum. Während in der französischen Literatur die Inkompatibilität mit dem bisherigen zeitintensiven HA-Arbeitsmodell betont wird, sieht die Schweizer Literatur den weiblichen, Teilzeitarbeitenden HA als künftig tragenden Pfeiler der Grundversorgung. Bezüglich sozialer Selektion finden in Frankreich zwei Arbeiten einen Zusammenhang zwischen soziodemografischem Hintergrund und HA-Mangel: das Medizinstudium selektioniere Studenten aus höheren Gesellschaftsschichten, und deren Suche nach „Wegen der Exzellenz" führe sie nicht in die HA-Medizin. In der Schweiz findet sich kein derartiger (statistisch signifikanter) Zusammenhang.

- Medizinische Sozialisation: Während auch in der Schweiz kritisiert wird, dass die Spitzenmedizin der Spezialisten zu sehr im Fokus von Fachwelt und Öffentlichkeit steht, wurden in Frankreich nicht nur die Spezialisten zur Elite erhoben, sondern die HA-Medizin auch jahrelang entwertet. Reformen in den 1950er und 1980er Jahren schufen einen Dualismus zwischen Spezialisten und HA (Spezialisten nur an Universitätskliniken ausgebildet, Zutritt nur durch Bestehen einer Prüfung, alle die nicht bestehen oder nicht antreten werden $\mathrm{HA}$ ) und verhalfen den Spezialisten durch Selektionslogik und längere Ausbildungsdauer zu einer Aura von medizinischer Exzellenz. Eine Abwendung von der HA-Medizin erfolgte über Jahre aus dem Bedürfnis heraus, der Elite anzugehören. Beim Erhalten dieses Dualismus spielen die klinischen Dozenten bis heute eine wichtige Rolle: selbst Teil der erfolgreichen Elite, stellen sie die HA-Medizin als Disziplin der Durchgefallenen dar. In der Folge geben 65\% der Assistenzärzte in HA-Medizin in einer aktuellen Umfrage an, dass die Entwertung der HA-Medizin als Demotivator bei ihrer Wahl wirke. Seit Einführung der ECN im Jahr 2004 gilt die HA-Medizin zwar als gleichgestellte Spezialität, im Wahlverhalten der Studenten bildet sich die alte Hierarchie jedoch weiterhin ab. Die Bedeutung eines Fachrichtung-bezogenen Wertesystems hat indessen zugenommen, weil bereits am Ende des 6 . Studienjahrs die definitive Fachrichtungswahl getroffen werden muss. In Frankreich beschreiben Arbeiten zudem einen Zusammenhang zwischen der Entwertung der HA-Medizin und der langjährigen Dominanz des biomedizinischen Modells. Dieses reduziert 
medizinische Probleme auf die Zell- und biomolekulare Ebene und blendet die in der HA-Medizin wichtige psychosoziale Ebene aus. Studenten finden in der Folge in der HA-Praxis nicht vor, worauf sie trainiert sind.

○ Weiterbildung: Im Gegensatz zu Frankreich verfügt die Schweiz bisher nur vereinzelt über einheitlich strukturierte, institutionell organisierte

Weiterbildungsangebote im Sinne eines von HA-Medizin-Instituten betreuten Curriculums. So wird als hemmender Faktor in der Wahl zum HA die Tatsache angeführt, dass im Gegensatz zu anderen Fachrichtungen die fünfjährige Weiterbildung selbst zu organisieren ist - teilweise unter finanziellen, zeitlichen oder geografischen Opfern. Die fehlende organisatorische und finanzielle Planungssicherheit wirke abschreckend. Zudem wird bemängelt, dass die theoretischen Weiterbildungsinhalte in der Schweiz wenig auf die HA-Medizin ausgerichtet sind. In Frankreich, wo Weiterbildungs-Curricula mit 6-monatigen Rotationen und 200 Stunden HA-Medizin-Theorie der landesweite Standard sind, findet die Kritik der Assistenzärzte auf einer anderen Ebene statt: Sie fordern mehr psychosoziale Schulung und Vorbereitung auf die Praxistätigkeit im Rahmen der theoretischen Blöcke und eine Verlängerung der dreijährigen Weiterbildungszeit auf vier Jahre, um sich eine grössere und breitere klinische Erfahrung anzueignen. In beiden Ländern herrscht ein Mangel an Praxisassistenz-Stellen. Dabei zeigen französische Arbeiten, dass gute (d.h., strukturierte, evidence based medicinegeleitete) Lehr-HA die Assistenzärzte motivieren, als Grundversorger tätig zu werden. Schlechte Lehrärzte hingegen schrecken von der Praxistätigkeit ab.

\section{Ü1: Diskussion}

Der systematische Vergleich der Literaturen beider Länder zeigte, dass in einer Mehrheit der Kategorien gleiche Probleme beschrieben werden, die französische Literatur aber häufig weiterführendes Wissen liefert, welches möglicherweise auf die Schweiz übertragbar ist. Anhand des systematischen Literaturvergleiches kristallisierten sich drei zentrale Themenkreise heraus:

\section{Ü2: Attraktive Arbeitsinhalte in unattraktiven Arbeitsstrukturen: Die Gruppenpraxis,} eine mögliche Lösung für das Problem?

Ein zentrales Problem ist die Tatsache, dass viele Nachwuchsmediziner die Arbeitsinhalte der HA-Medizin anziehend [38-44], die Arbeitsstrukturen in der HA-Medizin gleichzeitig jedoch abschreckend finden [6, 36, 38, 41, 45, 46]. Französische Autoren zeichnen die Problementstehung nach: Schweyer [44] berichtet von einer Neudefinition des Berufsmodells 
aus dem Privatleben heraus. Veränderte Geschlechts-, Paar- und Familiensoziologie verursachen ein Bedürfnis nach verringerter und besser steuerbarer Arbeitszeit. Dieses kann in einer Gruppenpraxis einfacher umgesetzt werden. Bloy [36] berichtet von einer neuen Individualsoziologie, die von Flexibilität und dem Ausprobieren von Möglichkeiten geprägt ist. Dies bedingt eine ökonomische Ungebundenheit, welche sich in eine Ablehnung des alten HA-Modells (grosse Investitionen in Einzelpraxis) übersetzt [47]. Hinzu kommt die für beide Länder beschriebene Angst vor Festlegung der Nachwuchsmediziner [36, 39, 44] und die durch französische Autoren damit in Zusammenhang gebrachte, durch Van de Velde [37] in mehreren europäischen Ländern nachgewiesene Norm unter jungen Erwachsenen, Engagement aufzuschieben. Als Lösung erscheinen das Teilen der Investitionskosten in einer Gruppenpraxis oder das Angestelltenverhältnis. Baude [48] und Abholz [49] beschreiben, wie Aus- und Weiterbildung die Jungmediziner auf die Arbeit in Team und Rücksprache mit Experten konditioniert. Die Jungmediziner lernen, dass Probleme vielgliedrig sind und möchten Verantwortung nur für den Teil übernehmen, für den sie mit ausreichend Wissen ausgestattet sind. Dies fördert den Wunsch, in einem pluridisziplinären Team zu arbeiten, z.B. in einer interdisziplinären Gruppenpraxis. Die daraus resultierende Spannung kann als Triebfeder für die in beiden Ländern zu beobachtende Transition vom Einzelpraxismodell zum Gruppenpraxismodell interpretiert werden. Im Ideal der Gruppenpraxis findet diese Teilursache des HA-Mangels zunehmend ihre Auflösung. In Frankreich könnten interdisziplinäre Gruppenpraxen sogar das Problem der „medizinischen Wüsten“ Iösen: gemäss einer nationalen Umfrage [50] geben 70\% der Internes an, dass Hilfe bei der Schaffung einer interdisziplinären Gruppenpraxis sie zur Niederlassung in ländlichen Regionen bewegen könnte.

Gerade bei dieser Transition der Praxisform ist aber Vorsicht geboten, will man die Arbeitsstruktur als Teilursache des HA-Mangels möglichst nachhaltig verbessern: eine steigende Anzahl an jungen HA beginnt nämlich ihre Arbeit im Angestelltenverhältnis in einer kommerziellen Gruppenpraxis, um ihre ökonomische Ungebundenheit zu wahren. Dabei drängt sich jedoch die Frage auf, ob dadurch nicht eine der meistgeschätzten Eigenschaften der HA-Medizin tangiert wird: die berufliche Autonomie. Mehrere französische Arbeiten [46, 51] erheben „Unabhängigkeit“ resp. „Autonomie“ als einen der wichtigsten Motivatoren für die Niederlassung unter den französischen HA-Anwärtern. Auch in der Schweiz bezeichnen die jungen Mediziner die Unabhängigkeit als positive und stark attraktive Strukturrealität der HA-Medizin [42, 52]. Es stellt sich die Frage: Welche ökonomische Organisationsform wird durch geringeres finanzielles Engagement den neuen Flexibilitätsansprüchen gerecht, wahrt aber zugleich die individuelle arbeits-inhaltliche Unabhängigkeit? Es gibt bereits ein paar Organisationsmodelle, die die Wahrung dieses Gleichgewichtes adressieren: 1.) Teilen der Investitionen in einer Gruppe von Ärzten. In der 
Folge bestehen weniger grosse finanzielle Zwänge durch problemlosere

Anfangsfinanzierung, Risikostreuung und Rationalisierung der Infrastruktur. 2.) Übernahme der Investitionskosten durch einen Dritten, dessen Investitionsmotiv nicht der Profit sondern die Sicherstellung der Grundversorgung darstellt. Ein Beispiel ist die Einrichtung und Vermietung der kompletten Praxisinfrastruktur an HA durch Gemeinden [39, S.126]. Damit entfallen für den jungen HA die Probleme der Anfangsfinanzierung und der Standortgebundenheit. Gleiches gilt für den Fall, dass eine entsprechend konstituierte Genossenschaft als Eigentümerin der Praxisinfrastruktur auftritt [53].

\section{Ü2: Die medizinische Sozialisation spielt eine doppelte Schlüsselrolle}

Verschiedene Arbeiten weisen darauf hin, dass der medizinischen Sozialisation eine wichtige Rolle bei der Erklärung des HA-Mangels zukommt. Unter medizinischer Sozialisation versteht man „die Gesamtheit aller Prozesse, mittels derer Neulinge/Neumitglieder (der Ärzteschaft) die in ihr geltenden Normen, Wertesysteme, Sprache etc. übernehmen, um ihr anzugehören“ [54]. Darunter fällt auch das - eventuell nur implizite - Ausbilden einer Meinung darüber, ob HA-Medizin etwas Spannendes ist und HA wichtige, interessante Arbeit leisten, oder eben nicht. Französische Arbeiten geben Hinweise darauf, dass sich das Resultat dieser Prägung mindestens auf zwei Arten auswirkt: 1.) Prägung des Einzelnen in Bezug auf die von inm geschätzten Arbeitsinhalte. Dies entscheidet darüber, ob ein Student seine Ansprüche in der HA-Medizin erfüllt sieht und sie attraktiv findet. 2.) Prägung des Ärztekollektivs in ihrer Wertschätzung der HA-Medizin als Bestandteil des Gesundheitswesens und als medizinische Fachdisziplin. Dies entscheidet über den Status der HA zumindest in der Mediziner-Community. Mit diesem Wissen drängen sich folgende Fragen auf: Wie und wann entstehen genau die Wertesysteme, anhand derer Medizinstudenten und Ärzte den persönlichen und gesellschaftlichen Wert der HA-Medizin bemessen? Wie könnte man das beeinflussen? Einige französische Autoren diskutieren dabei Inhalte, die auch in der Schweiz für die medizinische Sozialisation während des Studiums zutreffen könnten: $\underline{\text { Schweyer [44] hält fest, dass die Medizinstudenten bereits vor }}$ Studienbeginn eine „Repräsentanz" von der Hausarztmedizin aufbauen - durch persönliche Erfahrungen und Übernahme von gesellschaftlichen Diskursen [44, S.118]. Halter [55, S.471] beschreibt, dass im Jahre 1977 zwei Drittel der Medizinstudenten zu Beginn des Studiums, aber nur noch ein Fünftel der Studenten am Ende des Studiums in die HA-Praxis gehen wollten. Gleichzeitig sticht bBei der Betrachtung z.B. der Nachwuchsmediziner-Kohorte von Buddeberg et al. [57] sticht ins Auge, dass gerade mal 7\% der 715 betrachteten Medizinabsolventen des Abschlussjahres-2001 die HA-Medizin als Facharztwahl angaben. Bei ca. $40 \%$ Unentschlossenen hatten sich etwa $53 \%$ der Studenten bei Studienende gegen die HA Medizin entschlossen. Das Studium scheint sich demnach als zentraler Abschnitt bei 
der Nicht-Hervorbringung von HA hervorzutun. Schweyer [44] beschreibt die Dominanz der spezialisierten hochtechnisierten Medizin im universitären Umfeld, die mittels herausfordernder Interventionen oft den „Königswegs der Heilung“ begeht. Die Ausführung solcher heroisch anmutender Akte bietet die HA-Medizin dann in den Augen der Studenten nicht. Bloy [31] führt die Dominanz des biomedizinischen Modells in der Medizin des 19. und 20. Jahrhunderts an, welches die in der HA-Medizin häufiger in Erscheinung tretende psychosoziale Ebene ausblende. Die Studenten messen ihr folglich wenig Bedeutung zu und somit auch einem Teil des hausärztlichen Handelns.

Mit diesem Wissen drängen sich folgende Fragen auf: Wie entstehen genau die Wertesysteme, anhand deror Medizinstudenten und Ärzteden persönlichen und

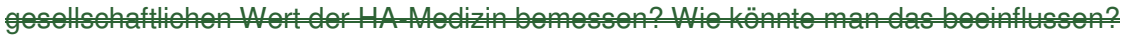
Einige Arbeiten geben Hinweise darauf, dass dabei das "Wann" beachtet werden muss: VorStudium: Schweyer [44] hält fest, dass die Medizinstudenten bereits vor Studienbeginn eine „Repräsentanz" von der Hausarztmedizin aufbauen - durch persönliche Erfahrungen und Übernahme von gesellschaftlichen Diskursen [44, S.118]. Halter [55] beschreibt eine Umfrage aus dem Jahre 1977, „in der zwei Drittel der [...] Medizinstudenten zu Beginn des Studiums, aber nur noch ein Fünftel der Studenten am Ende des Studiums in die HA Praxis gehen wollten." [55, S.471] Im gleichen Artikel berichtet Halter, dass heutzutage zu Studienbeginn gerade mal 3,8\% der Medizinstudenten die HA-Praxis als Berufswunsch angeben (zu Studienende dann ca. 10\%.). Welche Entwicklungen bewirkten diesen „vorstudentischen" Attraktivitätsschwund, der unabhängig scheint von tieferen Einblicken in die Medizin? Studium:-Tandjung [56] stellt fest, dass 54\% der frisch niedergelassenen HA ihre Berufsentscheidung während der Assistenzzeit (=Weiterbildung) getroffen haben. Daraus wird gefolgert, dass dieser Abschnitt die wichtigere Rolle bei der Hervorbringung von HA spielt als das Studium. Dies ist für die betrachtete Population (werdende HA) sicherlich richtig. Gleichzeitig sticht bei der Betrachtung z.B. der Nachwuchsmediziner-Kohorte von

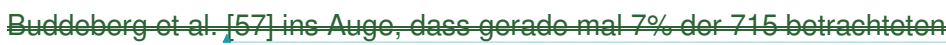
Medizinabsolventen des Abschlussjahres 2001 die HA-Medizin als Facharztwahl angaben. Beica, $40 \%$ Unentschlossenen hatten sich $53 \%$ der Studenten beiStudienende gegen die HA Medizin entschlossen. Das Studium scheint sich demnach als zentraler Abschnitt bei

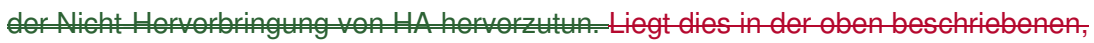
erdrückenden Dominanz der hochspezialisierten, biomedizinischen Sichtweise begründet? Wie könnte man ihr mehr „Menschenmedizin“ zur Seite stellen, diese erfahrbar machen? Beruf: Abholz [49] stellt fest, dass die Bevorzugung der "Spezialistenlogik" gegenüber der "Generalistenlogik" selbst im Berufsalltag des HA anzutreffen sei. So werde z.B. beider Qualitätssicherung in HA-Praxen häufig nur das "Spezialistisch-Eindimensionale“ bewertet - 
wie z.B. der durchschnittliche HbA16-Wert aller in der Praxis behandelter Diabetiker-,

\section{Ü2: Die weitreichende Bedeutung der Weiterbildung: spezifische Curricula von angemessener Dauer sind ein Muss}

Der systematische Literaturvergleich macht deutlich, dass das Schweizer

Weiterbildungsmodell mit seiner organisatorischen und finanziellen Planungsunsicherheit viel vom französischen Pendant lernen kann - vor allem im Hinblick auf den institutionellen Rückhalt der Weiterzubildenden in den französischen Instituten für HA-Medizin. Neben dieser wichtigen Frage nach der Organisation der Weiterbildung wird in beiden Ländern aber auch ihre „Ausstattung“ diskutiert und problematisiert: Während in der Schweiz die Weiterbildungsinhalte als wenig HA-Medizin-spezifisch beschrieben werden [58], wird aus Frankreich von einem Bedürfnis nach einer längeren und breiteren Weiterbildung berichtet $[59,60]$. Wie relevant die inhaltliche und zeitliche Ausstattung ist, zeigt sich in möglichen Zusammenhängen mit anderen Kategorien des HA-Mangels: In beiden Ländern geben Medizinstudenten etwa in der Kategorie „Arbeitsinhalte“ an, dass sie das breite Krankheitsspektrum der HA-Medizin abschrecke wegen der grossen Verantwortung und der assoziierten Unmöglichkeit, breitflächig kompetent zu sein [39, 61, 62]. Eine Weiterbildung, die dafür bekannt ist, dass sie breit ausbildet und Konzepte für den Umgang mit Unsicherheiten an die Hand gibt, könnte diese arbeitsinhaltlichen Demotivatoren entschärfen. Ein Zusammenhang mit der medizinischen Sozialisation stellt Buddeberg [40] her, indem eine allfälligen Reduktion der hausärztlichen Weiterbildungsdauer in der Schweiz auf drei Jahre einer Degradierung der HA-Medizin gleichgestellt wird. Hier wechselwirkt die Weiterbildungsdauer mit dem Ansehen der HA: Nur eine umfassende Weiterbildung kann die Ansicht hervorbringen, dass HA top ausgebildete Fachärzte mit eigenem Spezialwissen sind. Solch eine Ansicht kann dann auch Eingang in die medizinische Sozialisation finden und bereits früh an nachkommende Medizinergenerationen weitergegeben werden. In Hinblick auf eine solche Wirkung eines umfassenden Weiterbildungsprogrammes kann Frankreich von der Schweiz lernen: die gleiche Dauer der hausärztlichen Facharztausbildung wie die anderer Spezialitäten kann als Grund mitangeführt werden, warum die Schweizer HA nicht derart als „zweitklassige Mediziner“ stigmatisiert wurden wie in Frankreich.

Für die künftige Verbesserung der Weiterbildung bieten sich folgende Aussichten: In der Schweiz wurde die Notwendigkeit von HA-Medizin-spezifischen Curricula mit organisierten klinischen Rotationen erkannt und punktuell eingeführt, noch ist ihre Anzahl aber sehr gering [63, S.848]. Zudem sind wie Tandjung [63] skizziert für ihre weitere Etablierung wichtige Aufgaben zu lösen, wie z.B. deren nationale Vereinheitlichung zwecks grösserer Mobilität (bislang ist die Teilnahme an einem HA-Medizin-Curriculum meist an eine Niederlassungspflicht im Anbieter-Kanton geknüpft, bei Vereinheitlichung unter den Kantonen könnte sich diese Bestimmung erübrigen). Im Hinblick auf die HA-Spezifität der 
Weiterbildung bieten ebenfalls die Curricula einen vielversprechenden Gestaltungsraum, der die Umsetzung eines Lernzielkatalogs, wie er ihm Rahmen des neuen Facharzttitels für „Allgemeine Innere Medizin“ besteht, viel besser ermöglicht. Für die Qualität der hausärztlichen Weiterbildung in der Schwoiz gilt es schliesslich, allfällige Verkürzungsideen abzuwenden - nicht zu Letzt aufgrund der negativen Auswirkungen, die eine Reduktion der Weiterbildungsdauer im Gesamtsystem „HA-Mangel“ haben kann. In Frankreich scheint sich die Organisation der Weiterbildung auf einem guten Niveau zu befinden. Im Hinblick auf die inhaltliche Ausstattung kommt die Sprache häufig auf die Dauer der Weiterbildung: Eine breitere und reichere Weiterbildung scheint für viele nur durch deren Verlängerung erreichbar. Laut Angot [59] befürworten dies knapp 60\% der angehenden HA. Aktuell scheint aber eine Verlängerung von aktuell drei auf neu vier Jahre bis auf weiteres vom standespolitischen Diskussionstisch zu sein. In Frankreich bestehen zudem Vorschläge, an Universitätsspitäler angegliederte Notfallpraxen/Ambulatorien durch die Institute für HAMedizin zu betreiben und somit für deren Lehrtätigkeit zu erschliessen [48]. Solche Services universitaires de médecine générale ambulatoire könnten das in der HA-Medizin typische Patientengut an die Universitätsspitäler holen und somit bereits für Studenten - angeleitet durch erfahrene HA - teilweise erfahrbar machen.

\section{Ü2: Stärken und Schwächen}

Die Stärken dieser Arbeit liegen in ihrer Breite und Systematik: Kaum eine andere Arbeit in der gesichteten schweizerischen oder französischen Literatur versuchte eine anschauliche Übersicht über die bekannten Ursachen des HA-Mangels zu geben. Sie unternimmt einen systematischen Vergleich zwischen zwei Ländern über möglichst viele Bereiche, anstatt einen Vergleich eines Bereichs über möglichst viele Länder. Nach den Arbeiten zum Schweizer HA-Mangel wurde in den Suchmaschinen auf Deutsch gesucht, daher fanden praktisch ausschliesslich deutschsprachige Arbeiten Eingang in den Vergleich. Ein weiterer Selektionsbias liegt in der dritten Stufe der Literatursuche begründet, nämlich im Durchsuchen der Literaturverzeichnisse der als Treffer gewerteten Arbeiten: Bestimmte Arbeiten und Autoren tauchen darin als immer wiederkehrende Referenzpunkte auf und verdrängen dabei möglicherweise andere Arbeiten. Des Weiteren stellt die Arbeit keine eigentliche systematic review dar, beinhaltet also keine systematische Literaturauswertung, in welcher zum Beispiel auch die Zahl der ausgeschlossenen Arbeiten dargelegt werden müsste. 


\section{Ü1: Schlussfolgerungen und Ausblick}

Diese Arbeit zeigt konkrete Massnahmen auf, wie dem HA-Mangel aktiv entgegengewirkt werden kann:

- Veränderung der medizinischen Sozialisierung zu Gunsten der HA-Medizin

- Mehr Präsenz der HA-Medizin-spezifischen Inhalte im Studium

- national einheitlich strukturierte und organisierte Weiterbildung

- Genügend lange Weiterbildungszeit

- Förderung der Anpassung der Arbeitsstrukturen an veränderte Bedürfnisse (bei gleichzeitiger Wahrung der beruflichen Autonomie)

- Angleichung der Generalisten- an die Spezialistengehälter 


\section{Literaturverzeichnis}

1. Baicker K, Chandra A. Medicare spending, the physician workforce, and beneficiaries' quality of care. Health affairs. 2004;Suppl Web Exclusives:W4-184-97.

2. Ferrer RL, Hambidge SJ, Maly RC. The essential role of generalists in health care systems. Annals of internal medicine. 2005;142:691-9.

3. Macinko J, Starfield B, Shi L. The contribution of primary care systems to health outcomes within Organization for Economic Cooperation and Development (OECD) countries, 1970-1998. Health Serv Res. 2003;38:831-65.

4. Macinko J, Starfield B, Shi L. Quantifying the health benefits of primary care physician supply in the United States. International journal of health services : planning, administration, evaluation. 2007;37:111-26.

5. Burla L, Widmer M. Ärztinnen und Ärzte in der Schweiz: Bestand und Entwicklungen bis 2011. Neuchâtel: Schweizerisches Gesundheitsobservatorium OBSAN; 2012.

6. Tschudi P, Bally K, Zeller A. [Who wants to be a GP? A survey medical students and junior doctors]. Praxis. 2013;102:335-9.

7. Seematter-Bagnoud L, Junod J, Jaccard Ruedin H, Roth M, Foletti C, SantosEggimann B. Offre et recours aux soins médicaux ambulatoires en Suisse: Projections à I'horizon 2030. Neuchâtel: Schweizerisches Gesundheitsobservatorium OBSAN; 2008.

8. Bryman A. Documents as sources of data. Social Research Methods. Oxford: Oxford university press; 2004. p. 380-97.

9. Mayring P. Qualitative Inhaltsanalyse. Forum Qualitative Sozialforschung / Forum: Qualitative Social Research. 2000;1.

10. Kocher G, (Hrsg.). Gesundheitswesen Schweiz 2010-2012: eine aktuelle Übersicht. 4. ed. Bern: Huber; 2010. 464 p.

11. BFS. Gesundheitsstatistik 2012. Neuchâtel: Bundesamt für Statistik BFS; 2012.

12. Kraft E, Hostettler S. FMH-Ärztestatistik 2012. Schweizerische Ärztezeitung. 2013;94:453-7.

13. Jaccard Ruedin H, Roth M, Bétrisey C, Marzo N, Busato A. Offre et recours aux soins médicaux ambulatoires en Suisse. Neuchâtel: Schweizerisches

Gesundheitsobservatorium OBSAN; 2007.

14. Kraft E, Hersperger M. Auch dank Frauen und Ausländern ist die ärztliche Versorgung in der Schweiz garantiert. Schweizerische Ärztezeitung. 2011;92:1899901.

15. BFS. Das Medizinstudium in der Schweiz im Profil. Neuchâtel: Bundesamt für Statistik BFS; 2005.

16. Chevreul K. France health system review. Copenhagen: WHO Regional Office for Europe; 2010. 291 p.

17. Fauvet $L$. Les médecins au 1er janvier 2012. Série Études et résultats $n^{\circ} 796$. Paris: Direction de la recherche, des études, de l'évaluation et des statistiques, Ministère des Affaires sociales et de la Santé; 2012.

18. Fenina A. Les Comptes nationaux de la santé en 2010. Série Études et résultats $\mathrm{n}^{\circ}$ 773. Paris: Direction de la recherche, des études, de l'évaluation et des statistiques, Ministère des Affaires sociales et de la Santé; 2011.

19. ONDPS. Rapport 2006-2007, Tome 1: La médecine générale. Paris: Observatoire National de la Démographie des Professions de Santé (ONDPS); 2008. p. 151.

20. Barlet M, Collin C. Localisation des professionnels de santé libéraux. In: Brocas A-M, editor. Comptes nationaux de la santé 2009. Paris: Direction de la recherche, des études, de l'évaluation et des statistiques; 2010. p. 27-56.

21. Romestaing $P$, Le Breton-Lerouvillois $G$. Atlas de la démographie médicale en france: Situation au 1er janvier 2013. Conseil national de l'ordre des médecins CNOM, 2013.

22. Bloy G, Schweyer F-X, (ed.). Singuliers généralistes: Sociologie de la médecine générale. Paris: Presses de l'école des hautes études en santé publique; 2010. 
23. Attal-Toubert K, Vanderschelden M. La démographie médicale à l'horizon 2030: de nouvelles projections nationales et régionales, Série Études et résultats $n^{\circ} 679$. Paris: Direction de la recherche, des études, de l'évaluation et des statistiques, Ministère des Affaires sociales et de la Santé; 2009.

24. Fauvet $L$. Les affectations des étudiants en médecine à l'issue des épreuves classantes nationales en 2011, Série Études et résultats $n^{\circ}$ 802. Paris: Direction de la recherche, des études, de l'évaluation et des statistiques, Ministère des Affaires sociales et de la Santé; 2012.

25. Winckler M. L' "Examen National Classant" ou Comment pérenniser l'élitisme médical, "casser" les futurs soignants et détruire la médecine générale en France.2005. Available from: http://martinwinckler.com/spip.php?article655 [accessed 21.08.2013].

26. Liste et réglementation des diplômes d'études spécialisées de médecine. Arrêté du 22.09.2004. Paris: Ministère de l'éducation nationale, de l'enseignement supérieur et de la recherche; 2004.

27. Eco-Santé 2013: Les bases Eco-Santé en ligne [Internet]. Institut de recherche et documentation en économie de la santé. 2013. Available from: http://www.ecosante.fr [accessed 27.10.13]

28. OECD Health Data 2013 [Internet]. OECD. 2013. Available from: http://stats.oecd.org/Index.aspx?DataSetCode=HEALTH REAC [accessed 27.10.13].

29. Base de données de l'ocde sur la santé 2013: Donnés frequamment demandés [Internet]. OECD. 2013. Available from: http://www.oecd.org/fr/els/systemessante/basededonneesdelocdesurlasante2013donneesfrequemmentdemandees.htm [accessed 27.10.13].

30. Badertscher N, Schöni M, Zoller M, Rosemann T. Weiterbildung von angehenden Hausärzten an stationären Weiterbildungsstellen: Sicht der Chefärzte. Praxis. $2011 ; 100: 781-5$.

31. Bloy G. Une médecine générale durablement dépréciée? Enquête sur une évidence. In: ONDPS, editor. Rapport 2006-2007, Tome 1: La médecine générale. Paris: Observatoire National de la Démographie des Professions de Santé (ONDPS); 2008. p. 137-73.

32. Gaidioz C, Ruhlmann S. Pourquoi la spécialité médecine générale est-elle mal classée aux choix des épreuves classantes nationales? Thèse pour obtenir le grade de Docteur en Médecine. Lyon: Université Claude Bernard LYON I; 2008.

33. Hardy-Dubernet A-C. À propos d'une minute décisive: le choix d'une spécialité médicale. Journal de gestion et d'économie médicales. 2009;27:174-90.

34. Hardy-Dubernet A-C, Gadéa C. De «faire médecine» à «faire de la médecine». Document de travail. Paris: Direction de la recherche, des études, de l'évaluation et des statistiques, Ministère des Affaires sociales et de la Santé; 2005. p. 119-31.

35. Burch M-A, Cochard B, Corpataux N, Passaplan C, Rayroux C. Pénurie en médecins de premier recours dans le canton de Fribourg: Regard croisé des médecins généralistes établis dans le canton et des étudiants en médecine de Fribourg. Revue Médicale Suisse. 2013:1328-9.

36. Bloy G. Comment peut-on devenir généraliste aujourd'hui? Le renouvellement des médecins généralistes vu à travers une cohorte de jeunes diplômés. Revue française des affaires sociales. 2011:9-28.

37. Van de Velde C. Devenir adulte: sociologie comparée de la jeunesse en Europe. Paris: Presses universitaires de France; 2008.

38. Bismuth M. Pourquoi les internes choisissent-ils la médecine générale: La médecine générale revient dans les premiers choix. Médecine. 2013:136-41.

39. Buddeberg-Fischer B, Stamm M, Buddeberg C, Klaghofer R. Möglichkeiten zur Attraktivitätssteigerung der Hausarztmedizin aus der Sicht junger Ärztinnen und Ärzte. Gesundheitswesen. 2008;70:123-8. 
40. Buddeberg-Fischer B, Stamm M, Marty F. Attraktivitätssteigerung der Hausarztmedizin: Ansichten und Vorschläge von praktizierenden Hausärzten. Primary Care. 2007;7:639-41.

41. Burghardt L, Dreisbach M, Shahla H, Businger A. Swiss Student's Arguments in Favour and against a Career in Primary Care. Primary Health Care. 2012;2.

42. Hasler L, Stamm M, Buddeberg-Fischer B. Zukünftige Hausärztinnen und Hausärzte: Gründe für die Berufswahl und berufliche Kernkompetenzen. Praxis. 2008;97:127785.

43. Rouger A-S. La médecine générale: un choix par défaut aux épreuves classantes nationales? Étude multicentrique sur la concrétisation des souhaits des étudiants aux ECN. Thèse pour le doctorat en médecine. Rouen: Université de Rouen; 2013.

44. Schweyer F-X. Souhaits et aspirations des médecins qui envisagent l'exercise de la médecine générale. In: ONDPS, editor. Rapport 2006-2007, Tome 1: La médecine générale. Paris: Observatoire National de la Démographie des Professions de Santé (ONDPS); 2008. p. 89-135.

45. Bundesrat. Strategie gegen Ärztemangel und zur Förderung der Hausarztmedizin: Bericht des Bundesrates in Erfüllung der Motion 08.3608 von Nationalrätin Jacqueline Fehr „Strategie gegen Ärztemangel und zur Förderung der Hausarztmedizin“ vom 2. Oktober 2008. Bern 2011.

46. ORS-Aquitaine. Motivations et freins à l'installation des médecins généralistes libéraux: synthèse de la littérature. Bordeaux: Observatoire régional de la santé d'Aquitaine, 2011.

47. Bloy G. La transmission des savoirs professionnels en médecine générale : le cas du stage chez le praticien. Revue Française des Affaires Sociales. 2005:101-25.

48. Baude N, Flacher A, Bosson J-L, Marchand O. Soins primaires: crise et dynamique d'avenir: Les attentes des internes de troisième cycle de médecine générale. Médecine. 2008:4:135-40.

49. Abholz H-H. Die Umerziehung der Allgemeinmedizin. ZFA-Zeitschrift für Allgemeinmedizin. 2007;83:85-8.

50. ISNAR-IMG. Enquête nationale sur les souhaits d'exercice des internes de médecine générale: résultats. Lyon: Inter syndicale nationale autonome représentative des internes de médecine générale, 2011.

51. Decorde A. Déterminants à l'installation en médecine générale: analyse croisée des médecins généralistes remplaçants et jeunes installés du sécteur nord alpin. Thèse pour l'obtention du doctorat en médecine. Grenoble: Université Joseph Fourier; 2011.

52. Buddeberg-Fischer B, Stamm M, Marty F. Family medicine in Switzerland: training experiences in medical school and residency. Family Medicine. 2007;39:651-5.

53. Rohner A. Die Genossenschaftspraxis: aus dem Dorf und für das Dorf. Primary Care 2012;12:394-5.

54. Hafferty F. Medical School socialisation. In: Ritzer G, editor. Blackwell Encyclopedia of Sociology: Wiley-Blackwell; 2007.

55. Halter U, Tschudi P, Bally K, Isler R. Berufsziel von Medizinstudierenden. Primary Care. 2005;5:468-72.

56. Tandjung R, Senn O, Marty F, Kraussa L, Rosemann T, Badertscher N. Career after successful medical board examination in general practice: a cross-sectional survey. Swiss Medical Weekly. 2013;143.

57. Buddeberg-Fischer B, Klaghofer R, Abel T, Buddeberg C. Swiss residents' speciality choices: impact of gender, personality traits, career motivation and life goals. BMC health services research. 2006;6.

58. Badertscher N, Schöni M, Berger L, Zoller M, Rosemann T. [Vocational training of future GPs in Swiss hospitals: the view of assistant physicians]. Praxis. 2011;100:349-55.

59. Angot $O$. Faut-il réformer l'internat de médecine générale? La revue du practicien médecine générale. 2007;21:633-4. 
60. Franco Rodrigues A. Représentations et conceptions de la médecine générale: regards croisés entre internes et médecins généralistes. Thèse pour le diplôme d'état de docteur en médecine. Paris: Université Pierre et Marie Curie (Paris 6); 2012.

61. Buddeberg-Fischer B, Klaghofer R, Stamm M. Family Physicians in Switzerland: Transition From Residency to Family Practice. Family Medicine. 2011;43:29-36.

62. Navarro L, Dubois J-P. Désintérêt des étudiants de $6 e$ année pour la médecine générale: une étude auprès de 15 volontaires lyonnais. La revue du practicien médecine générale. 2007;21:466-7.

63. Tandjung R, Garaventa-Tadres D, Rosemann T, Djalali S. Spezifische Weiterbildungsangebote für Hausarztmedizin in der Schweiz: Bestandsaufnahme verschiedener kantonaler Programme. Praxis. 2013;102:843-9. 
Tabelle 1: Vergleich Eckdaten Gesundheitswesen Schweiz versus Frankreich

\begin{tabular}{|c|c|c|}
\hline & Schweiz & Frankreich \\
\hline Allgemein & 8 Mio. Einwohner & 65 Mio. Einwohner \\
\hline \multirow{3}{*}{$\begin{array}{l}\text { Politisches System } \\
\text { Gesundheitswesen (GW) } \\
\text { Koordination d. GW }\end{array}$} & $\begin{array}{l}\text { Föderale Republik mit stark ausgeprägtem } \\
\text { Föderalismus ( } 26 \text { Kantone, } 7 \text { Regionen) }\end{array}$ & $\begin{array}{l}\text { Republik mit starker zentralistischer } \\
\text { Tradition (101 Departm., } 26 \text { Regionen) }\end{array}$ \\
\hline & Typ Bismarck & Typ Bismarck mit Beveridge-Zielen \\
\hline & $\begin{array}{l}\text { Kantone betreiben Infrastruktur, Bund } \\
\text { zunehmend nationale Gesetzeskompetenz }\end{array}$ & $\begin{array}{l}\text { Gesundheitsministerium starke Kontrolle, } \\
\text { zunehmend regionale Bedarfsplanung }\end{array}$ \\
\hline Volkswirt. Bedeutung & 13.5\% der Gesamtbeschäftigung & 7.6\% der Gesamtbeschäftigung \\
\hline \multicolumn{3}{|l|}{ Versorgung } \\
\hline \multirow{3}{*}{$\begin{array}{l}\text { Allg. Ärztedichte } \\
\text { - nach Sektoren } \\
\text { - nach Regionen }\end{array}$} & 3.8/1'000 Einwohner & 3.4/1'000 Einwohner \\
\hline & $53 \%$ ambulant, $45 \%$ stationär & $59 \%$ ambulant, $34 \%$ stationär \\
\hline & $\begin{array}{l}\text { Ärztedichte liegt in } 4 \text { Kantonen um mehr } \\
\text { als } 30-50 \% \text { unter dem nationalen Mittel }\end{array}$ & $\begin{array}{l}\text { Massive Unterschiede zwischen den } \\
\text { Departementen (deserts médicaux) }\end{array}$ \\
\hline Feminisierung (\% Frauen) & $\begin{array}{l}\text { Ärzteschaft: } \quad 29 \%(2000) \rightarrow 37.5 \%(2012) \\
\text { Assistenzärzte: } 56 \%(2010)\end{array}$ & $\begin{array}{l}\text { Ärzteschaft: } \quad 30 \%(1990) \rightarrow 41 \%(2012) \\
\text { Assistenzärzte: } 60 \%(2010)\end{array}$ \\
\hline Ausländeranteil & $28 \%$ ( $40 \%$ stationär, $17 \%$ ambulant) & $9 \%$ \\
\hline \multirow{3}{*}{$\begin{array}{l}\text { Spitalbetten/1'000 E } \\
\text { Grundversorger-Dichte } \\
\text { Spezialisten-Dichte }\end{array}$} & 4.9 (3.0 Akut) & 6.4 (3.4 Akut) \\
\hline & 0.9/1'000 Einwohner & 0.8/1'000 Einwohner \\
\hline & 1.1/1'000 Einwohner & 0.9/1'000 Einwohner \\
\hline \multicolumn{3}{|l|}{ Fokus Hausarztmedizin } \\
\hline \multirow[t]{3}{*}{ Praxis-Grundausrüstung } & $\begin{array}{l}\text { Untersuchungsraum \& Instrumente, EKG } \\
\text { Basislabor und Röntgen i.d.R. in Praxis }\end{array}$ & $\begin{array}{l}\text { Untersuchungsraum \& Instrumente, EKG } \\
\text { Basislabor und Röntgen i.d.R. extern }\end{array}$ \\
\hline & $\begin{array}{l}\text { Bestehend, } \\
\text { Verschlechterung bevorstehend }\end{array}$ & $\begin{array}{l}\text { Bestehend, } \\
\text { Verschlechterung bevorstehend }\end{array}$ \\
\hline & $\begin{array}{l}\text { Umverteilung [stat } \leftarrow \text { ambulant }(\text { Spez } \leftarrow \text { HA)] } \\
\text { Konzentration der HA auf urbane Gebiete }\end{array}$ & $\begin{array}{l}\text { Umverteilung [stat } \leftarrow \text { ambulant }(\text { Spez } \leftarrow \text { HA)] } \\
\text { Konzentration der HA auf urbane Gebiete }\end{array}$ \\
\hline \multicolumn{3}{|l|}{ Ausblick 2030} \\
\hline \multirow{4}{*}{$\begin{array}{l}\text { Angebot Hausärzte } \\
\text { Nachfrage Hausärzte } \\
\text { Angebot Spezialisten } \\
\text { Nachfrage Spezialisten }\end{array}$} & sinkend ( 21 Mio. Konsult. $\rightarrow 13$ Mio) & sinkend \\
\hline & steigend (21 Mio. Konsult. $\rightarrow 23$ Mio.) & steigend \\
\hline & konstant & sinkend \\
\hline & konstant & {$[\mathrm{KA}]$} \\
\hline \multicolumn{3}{|l|}{ Ärztlicher Nachwuchs } \\
\hline \multirow{4}{*}{$\begin{array}{l}\text { Dauer \& Aufbau } \\
\text { Eintrittsselektion } \\
\text { Anz. Studienabgänger } \\
\text { Wahl der Spezialität }\end{array}$} & $\begin{array}{l}6 \mathrm{~J} \text {. Ausbildung, 5-6 J. Weiterbildung } \\
\text { Mindestdauer Weiterbildung HAM: } 5 \mathrm{~J} \text {. }\end{array}$ & $\begin{array}{l}6 \mathrm{~J} \text {. Ausbildung, 3-5 J. Weiterbildung } \\
\text { Mindestdauer Weiterbildung HAM: } 3 \mathrm{~J} \text {. }\end{array}$ \\
\hline & Eignungstest oder 1. Propädeutikum & Gymnasiale Bildung + 1. Propädeutikum \\
\hline & $743(2011)$ & $6^{\prime} 976(2011)$ \\
\hline & $\begin{array}{l}\text { Freie Bewerbung } \\
\text { (Interesse, Noten, Kontakte) }\end{array}$ & $\begin{array}{l}\text { Zuteilung (épreuves classantes nationales) } \\
\text { (Wahl in Reihenfolge der Rangliste) }\end{array}$ \\
\hline \multicolumn{3}{|l|}{ Kosten \& Finanzierung } \\
\hline \multirow{3}{*}{$\begin{array}{l}\text { Gesamtkosten } \\
\text { Wachstum in \% } \\
\text { Verrechnungsart }\end{array}$} & 62.5 Mia. CHF (10.9\% des BIP) & 234.1 Mia. Euro (12.1\% des BIP) \\
\hline & $3.8 \%$ ( $\varnothing$ seit 1995, Tendenz fallend) & $5.3 \%$ ( $\varnothing$ seit 1995, Tendenz fallend) \\
\hline & $\begin{array}{l}\text { Ambulant: Einzelleistungsvergütung } \\
\text { Stationär: Fallpauschalen }\end{array}$ & $\begin{array}{l}\text { Ambulant: Einzelleistungsvergütung } \\
\text { Stationär: Fallpauschalen }\end{array}$ \\
\hline \multirow{2}{*}{$\begin{array}{l}\text { Leistung für Kosten } \\
\text { Finanzierung }\end{array}$} & EHC-Index 2012: Rang 7/34 & EHC-Index 2012: Rang 8/34 \\
\hline & 19\% Staat, 42\% SozVersich., 25\% Privat & $76 \%$ SozVers., $14 \%$ PrivatVers., $10 \%$ Privat \\
\hline Versicherungsart & Obligatorische Grundversicherung & Obligatorische Grundversicherung \\
\hline Kosten pro Person & $\begin{array}{l}\text { +/- einheitliche Kopfprämie, } \\
\text { einkommensabh. Prämienverbilligung }\end{array}$ & Einkommensabhängige Prämie \\
\hline $\begin{array}{l}\text { Zugang zum } \\
\text { Gesundheitswesen }\end{array}$ & $\begin{array}{l}\text { Mit Grundversicherung freie Ärzte-Wahl } \\
\text { im ambulanten Bereich. Viele wählen } \\
\text { jedoch Versich.Modelle mit Triagierung. }\end{array}$ & $\begin{array}{l}\text { Mit Grundversicherung Zugang praktisch } \\
\text { nur zu Ärzten im Sektor } 1 \text { (meiste HA, aber } \\
\text { wenig Spezialisten). }\end{array}$ \\
\hline Abrechnung ambulant & Tiers garant & Tiers garant \\
\hline Abrechnung stationär & Tiers payant & Tiers payant \\
\hline
\end{tabular}

${ }^{1}$ Die OECD definiert als Grenze der genügenden HA-Versorgung 1 HA/1'000 Einwohner. EHC: Euro Health Consumer Index 
Abbildung 1: Das Interaktionsmodell. Der Entscheid Hausarzt Ja/Nein findet in der Interaktion zwischen extrinsischen und intrinsischen Faktoren statt.

$\rightarrow$ [JPEG Interaktionsmodell im Bilder-Ordner]

Abbildung 2: Semiquantitativer Vergleich der Literatur beider Länder: Frankreich zeigt im Vergleich zur Schweiz eine intensivere Auseinandersetzung mit der Kategorie „Ausbildung Medizinische Sozialisation“. Die Schweiz zeigt im Vergleich zu Frankreich eine intensivere Auseinandersetzung mit den Kategorien „Weiterbildung" und „Ökonomie und Sozialstatus“ $\rightarrow$ [JPEG Semiquantitativer Vergleich im Bilder-Ordner] 


\begin{tabular}{|c|c|c|c|}
\hline Kategorie & FR & $\mathrm{CH}+$ & Synthese \\
\hline Arbeitsinhalte & $\begin{array}{l}\text { Grundsätzlich ziehen breites } \\
\text { Krankheitsspektrum und Arzt-Patienten- } \\
\text { Beziehung an. Doch werden genau sie von } \\
\text { anderen Studenten nicht geschätzt (Angst } \\
\text { vor Verantwortung, unerfüllbares } \\
\text { Kompetenzbedürfnis, Missfallen am } \\
\text { Verkleidet-Sein der medizinischen Inhalte in } \\
\text { emotionaleren Bekundungen). }\end{array}$ & $\begin{array}{l}\text { Breites Krankheitsspektrum und Arzt- } \\
\text { Patienten-Beziehung gehören zu stärksten } \\
\text { Argumenten für die HA-Medizin. Allerdings } \\
\text { wird gerade das breite Krankheitsspektrum } \\
\text { auch als Gefahr (Risiko, Fehler zu machen) } \\
\text { oder als Widerspruch zum } \\
\text { Kompetenzbedürfnis erlebt. }\end{array}$ & $\begin{array}{l}\text { Sowohl in Frankreich als auch in der Schweiz } \\
\text { gehören das breite Krankheitsspektrum und die } \\
\text { privilegierte Arzt-Patienten-Beziehung zu den } \\
\text { wichtigsten Motivatoren für die HA-Medizin. Das } \\
\text { breite Krankheitsspektrum schreckt jedoch einige } \\
\text { wegen der (zu) grossen Verantwortung und der } \\
\text { assoziierten Un-möglichkeit, breitflächig kompetent } \\
\text { zu sein, ab. }\end{array}$ \\
\hline $\begin{array}{l}\text { Arbeits- } \\
\text { strukturen }\end{array}$ & $\begin{array}{l}\text { Das neue Arbeitsideal (interdisziplinäre } \\
\text { Gruppenpraxis) deckt sich trotz sichtbarem } \\
\text { Transitionsvorgang noch nicht mit der } \\
\text { Arbeitsrealität der alten HA. Diese Realität } \\
\text { treffen die jungen Assistenzärzte in der } \\
\text { Praxisassistenz noch an und erleben sie als } \\
\text { abschreckend. }\end{array}$ & $\begin{array}{l}\text { Die (interdisziplinäre) Gruppenpraxis ist das } \\
\text { neue Ideal der Arbeitsbedingungen. Es gibt } \\
\text { Hinweise darauf, dass sich die Realität immer } \\
\text { noch von den Wünschen unterscheidet, } \\
\text { zumindest in der Wahrnehmung der } \\
\text { Studienabgänger. Neueste Trends zeigen ein } \\
\text { Angleichen der Realität an die Wünsche. }\end{array}$ & $\begin{array}{l}\text { Sowohl in Frankreich als auch in der Schweiz ist das } \\
\text { neue Arbeitsideal die (interdisziplinäre) } \\
\text { Gruppenpraxis. In beiden Ländern ist die Realität } \\
\text { aus Sicht der Studenten noch nicht so wie sie sich } \\
\text { diese wünschen, obwohl deutliche } \\
\text { Transitionsprozesse im Gange sind. }\end{array}$ \\
\hline $\begin{array}{l}\text { Ökonomie/ } \\
\text { Sozialstatus }\end{array}$ & $\begin{array}{l}\text { Der im Vergleich zu den Spezialisten } \\
\text { niedrigere HA-Lohn macht HA-Medizin } \\
\text { unattraktiv. } \\
\text { Investitionen und wiederkehrende Kosten im } \\
\text { Zusammenhang mit Praxiseröffnung und - } \\
\text { Betrieb wirken demotivierend, da sie zu einer } \\
\text { bestimmten Anzahl von Arbeitsstunden über } \\
\text { eine bestimmte Anzahl an Jahren zwingen. }\end{array}$ & $\begin{array}{l}\text { In der Schweiz persistiert weiterhin eine } \\
\text { Einkommensschere zwischen Spezialisten } \\
\text { und Allgemeinärzten. Der tiefere Lohn gehört } \\
\text { schon unter jungen Medizinstudenten zu den } \\
\text { häufigst genannten Demotivatoren der HA- } \\
\text { Medizin-Wahl. Das gleichbleibende } \\
\text { unternehmerische Risiko der Praxistätigkeit } \\
\text { bei niedrigerem Lohn wirkt abschreckend. }\end{array}$ & $\begin{array}{l}\text { Sowohl in Frankreich als auch in der Schweiz } \\
\text { verdienen niedergelassene Spezialisten tendenziell } \\
\text { mehr als niedergelassene Allgemeinmediziner, in FR } \\
\text { ist dieser Unterschied noch ausgeprägter. } \\
\text { Medizinstudenten und Assistenzärzte beider Länder } \\
\text { zählen dies zu den häufigsten Gründen gegen die } \\
\text { HA-Medizin-Wahl. }\end{array}$ \\
\hline Weiterbildung & $\begin{array}{l}\text { Grundsätzlich einheitlich strukturiert mit } \\
\text { national vorgegebenem Curriculum. } \\
\text { Unzufriedenheit herrscht bezgl. relativ kurzer } \\
\text { ambulanter Ausbildung, die theoretischen } \\
\text { Blöcke sind in den Augen der AA } \\
\text { anpassungswürdig (mehr psychosoziale } \\
\text { Schulung gewünscht). }\end{array}$ & $\begin{array}{l}\text { Trotz der grossen Bedeutung für den } \\
\text { Entscheid, HA zu werden, ist die } \\
\text { Weiterbildung in der Schweiz bislang relativ } \\
\text { beschwerlich: hoher organisatorischer } \\
\text { Aufwand, finanzielle, geografische oder } \\
\text { zeitliche Opfer. Sie ist zudem wenig HA- } \\
\text { Medizin-spezifisch. }\end{array}$ & $\begin{array}{l}\text { Im Gegensatz zu Frankreich ist die Weiterbildung } \\
\text { zum HA in der Schweiz weder institutionell } \\
\text { organisiert noch einheitlich strukturiert, ist wenig } \\
\text { HA-Medizin-spezifisch und der angehende HA muss } \\
\text { für eine ideale Berufsvorbereitung unter Umständen } \\
\text { eine längere Weiterbildungszeit, tieferen Lohn u./od. } \\
\text { häufigere Ortswechsel in Kauf nehmen. }\end{array}$ \\
\hline
\end{tabular}


HA-Medizin ist während dem Studium kaum präsent. Die wenigsten Studenten machen ein HA-Medizin-Praktikum. Spezialisierte, segmentierte Ausbildung bringe entsprechend veranlagte Mediziner hervor. Fehlen von betriebswirtschaftlichen Inhalten. "auserlesenen Spezialisten" und durchgefallenen $\mathrm{HA}$ " hervor. Medizinstudenten bauen bis heute ein Fachrichtung-bezogenes Wertesystem auf das zu Ungunsten der HA-Medizin-Wah funktioniert. Zu dieser Entwertung tragen medizinhistorische Gründe bei. (Dominanz d. biomedizinischen Modells)

\begin{tabular}{l|l} 
Privatleben & Die Berufstätigkeit der Frau verändert die
\end{tabular} Paarsoziologie -> Der Mann kann und will nicht mehr $150 \%$ arbeiten. Neues Primat des Familienlebens tradiert altes zeitintensives HA-Arbeitsmodell zusätzl.

\section{Biografie/ Feminisierung bringt neue Vorstellung bezgl.}

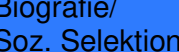
Gleichgewicht

Arbeit <-> Privatleben, die inkompatibel ist mit

Medizinstudium selektioniert v.a.

Intellektuellenkinder, dabei wählen gerade

Ärzte-Kinder selten HA-Medizin, Mittelklasse-

Kinder hingegen häufiger HA-Medizin.

\section{Psychische}

Motive
Gegen Niederlassung als HA sprechen...

- Angst vor vorzeitiger Verwurzelung

(„Norm der europäischen Jugend“)

- reaktives Freiheitsbedürfnis nach

Studium voller Systemzwänge.
Die HA-Medizin erscheint im Studium zeitlich und inhaltlich untervertreten.

Es fehlen betriebswirtschaftliche und gesundheitsökonomische Inhalte, die Unbehagen gegenüber eigenständiger Praxistätigkeit abbauen würden.

In medizinischer Fachwelt und Öffentlichkeit liege Fokus zu sehr auf der „Spitzenmedizin der Spezialisten. Im Studium erfahren die Studenten eine Geringschätzung der HAdem alten HA-Arbeitsmodell. Das

\section{Gegenüber früheren HA-Generationen} verschob sich bei jungen HA das

Gleichgewicht Arbeit <-> Privatleben stark zu Gunsten der Familie. Dies ist bei jungen Spezialisten weniger ausgeprägt.

\section{Die Feminisierung der Medizin wird als neue} Realität häufig erwähnt, die

Arbeitszeitvorstellungen der Frauen wirken aus.

In der schweizer Literatur finden sich keine bis wenig Hinweise auf den Einfluss des Wahl zum HA

An einer Niederlassung als HA hindern..

- Angst vor definitiver Festlegung durch
Sowohl in Frankreich als auch in der Schweiz ist die HA-Medizin im Studium zeitlich und inhaltlich untervertreten. In Frankreich ist dies noch deutlich ausgeprägter und verschlimmert um die Tatsache, dass das HA-Medizin-Praktikum/Tutorat weniger etabliert ist.

Im Gegensatz zur Schweiz besteht in Frankreich eine historisch gewachsene, durch Gesetze institutionalisierte Entwertung der HA-Medizin. Zusätzlich sind in Frankreich medizin-historisch Ursachen der Entwertung beschrieben. In beiden Ländern scheinen die Medizinstudenten eine Geringschätzung der HA-Medizin durch Dozenten und Spitalärzte zu erleben. sich auf die verfügbare totale Arbeitskapazität

soziodemographischen Hintergrundes bei der

Praxiseröffnung

Angst vor Verantwortung als HA
In beiden Ländern wird im Gleichgewicht Arbeit <-> Privatleben eine markante Verschiebung zugunsten des Privatlebens festgestellt. Diese tradiert das alte zeitintensive Arbeitsmodell des HA.

\section{Sowohl in Frankreich als auch in der Schweiz werden} die Feminisierung und ihre Auswirkungen

thematisiert. Im Gegensatz zur Schweiz finden sich in Frankreich Zusammenhänge zwischen soziodemografischem Hintergrund und HA-Mangel. Insbesondere die Überselektion aus gewissen Gesellschaftsschichten wirke sich nachteilig auf die Sicherstellung des HA-Nachwuchses aus.

Sowohl in Frankreich als auch in der Schweiz zeigen die jungen Ärzte eine Angst vor definitiver

Festlegung und vorzeitiger Verwurzelung. Dies hindert sie daran, eine Tätigkeit als niedergelassener HA aufzunehmen. 
Appendix 1: Semiquantitative Gewichtung der französischen Literatur

$\rightarrow$ [JPEG SemiquantGewichtung-FR im Appendix-Ordner]

Appendix 2: Semiquantitative Gewichtung der Schweizer Literatur

$\rightarrow$ [JPEG SemiquantGewichtung-CH im Appendix-Ordner]

Appendix 3: Ursachen des Hausärzte (HA)-Mangels nach Kategorien, Frankreich.

In Standardschrift HA-Mangel-Ursachen, in Kursivschrift wichtige Zusatzinformationen. Hervorhebungen fett. AA meint Assistenzärzte ungeachtet der Fachrichtung, MedG-AA bezeichnet Assistenzärzte in der Fachrichtung Médecine générale.

$\rightarrow$ [Ländertabelle FR samt Bibliographie im Appendix-Ordner]

Appendix 4: Ursachen des Hausärzte (HA)-Mangels nach Kategorien, Schweiz.

In Standardschrift HA-Mangel-Ursachen, in Kursivschrift wichtige Zusatzinformationen. Hervorhebungen fett. Wo nicht näher eingegrenzt steht AA für Assistenzärzte ungeachtet der Fachrichtung.

$\rightarrow$ [Ländertabelle CH samt Bibliographie im Appendix-Ordner]

Appendix 5 (ehemals Tabelle 2) : Qualitativer Vergleich der Ursachen des Hausärztemangels Frankreich versus Schweiz 
Extrinsische Faktoren

(äussere Realitatten)

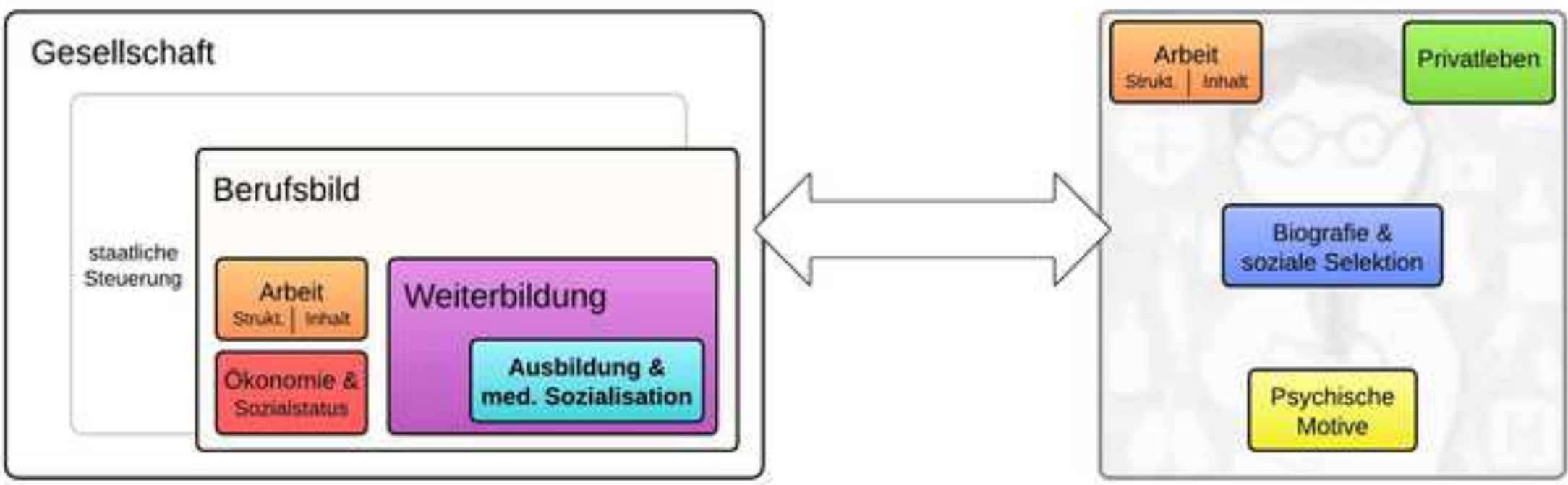

Intrinsische Faktoren

(Motivationen, Vorstellungen, Bedürnisse) 

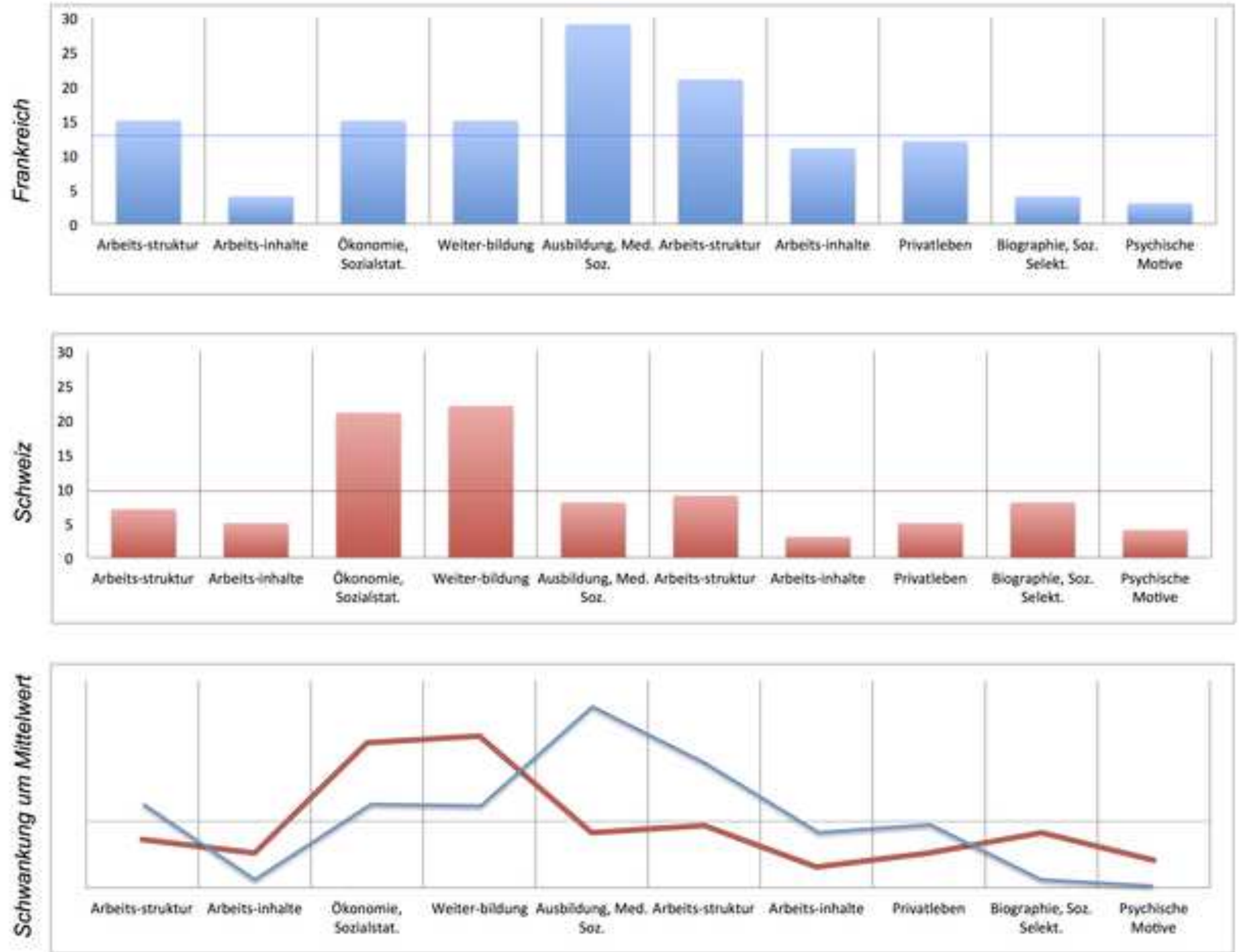
ARTKKELANGABEN

Autor Jahr Design

Angot 2007 Quantitativ

Augros. 2011 Quantitativ

Augros 2012 Quantitativ

Aulagnier et 2007 Quantitativ

Barlet et al. 2011 Quantitativ

Baude et al. 2008 Quantitativ

Bellamy 2010 Quantitativ

Bismuth et a 2013 Quantitativ

Bloy

Bloy

Boy

Bouton

Dacrur

2005 Qualitativ

2008 Qualitativ

2011 Qualitativ

Franco Rodri 2012 Qualitativ

GaidiozRuhir 2008 Quantitativ

Gallois et al. 2006 Mini-Review

Gaucher 2013 Qualitative

Hardy-Duber 2009 Qualitativ

Hardy-Dubet 2005 Qualitativ

ISNAR-EMG 2011 Quantitativ

Leferre et al. 2010 Quantitativ

Navarro 2007 Qualitativ

OnS Aquitair 2011 Mini-Review

- Rouger 2013 Quantitativ

Schweyer $2008 \mathrm{Min}+$ Review

Spiess

2013 Quantitativ
Decorde 2011 Quantitativ.

Iakoubovitet 2012 Quantitativ

2009 Aufsatz

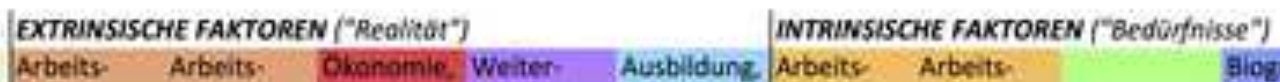

Interessens: Publikat. struktur

Sorialstat.

biidury

Med. Sor. struktut inhalte Privatieben Sor.Selekt Mative

Syndicat IMG Journal

AAMedG Dr.Art.

Regierung.

Regierung

AAMedG Journal

Regierung Série

Scholar Journal

Scholar Journal

Scholar

Scholar

Scholar

CNOM

AA MedG

AAMedG

AA MedG

Scholar

Ale

Scholat

Scholat

Syndicat IMG geritht

Regierung Série

Az

Scholat

Regierung

AAMed6

Scholar

AA MedG

Aktivist

Bericht

Journal

Journal

Bericht

Dr.Arb.

Dr.Arb.

Dr.Arb.

Journal

Joumal

Journal

Bericht

tournal

Journal

Bericht

De.Arb.

Bericht

Dr. Arb.

Journal
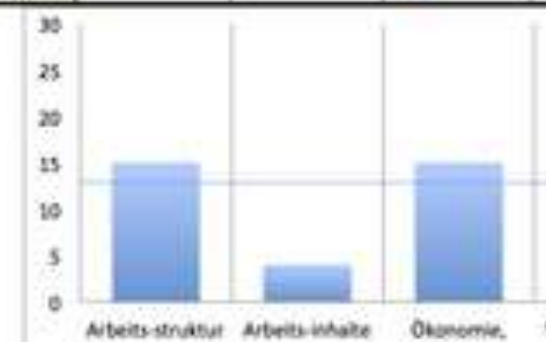

Obinomit.

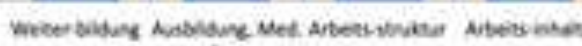

Sor.

o

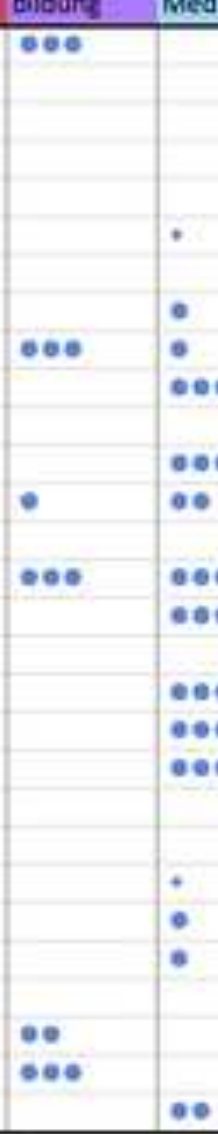

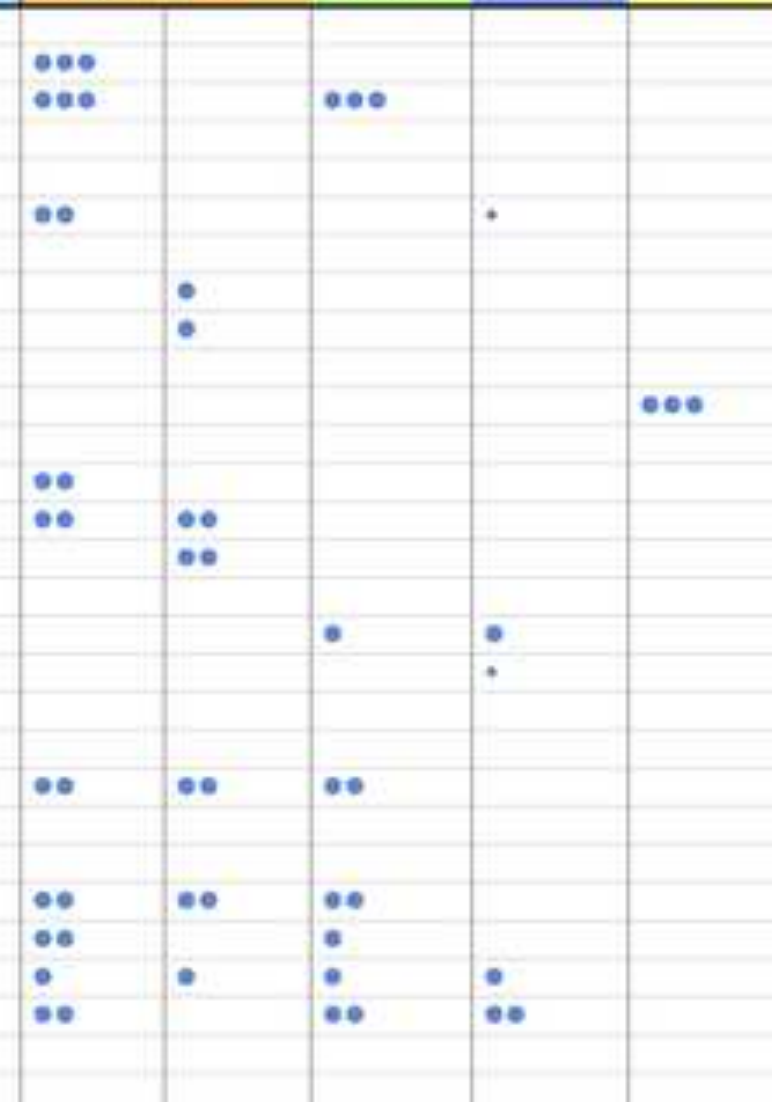

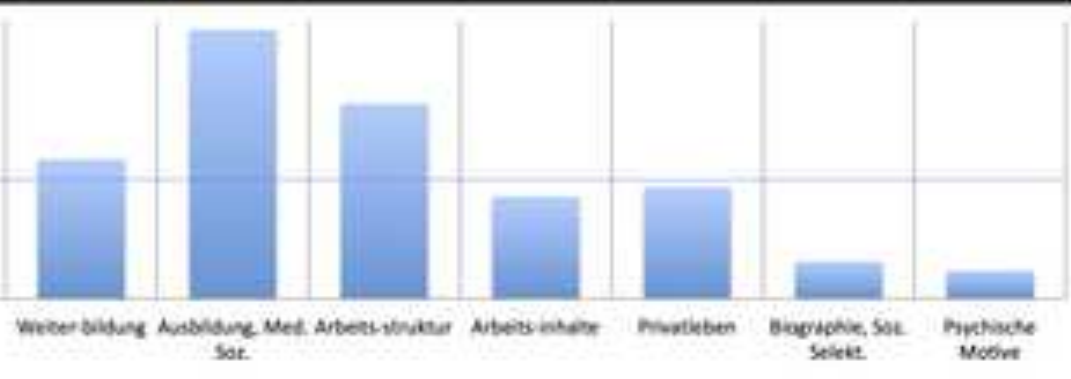

Legendet der Kategorie sind gewidmet..

- mind. 1 Absotz (Parogroph) oo mind. 1 Abschnitt (mit Oberschrift)

- Methrheir d. Arbeit "nicht is Forschungsfroge enthoken, aber ousfühlicher Kommentor in Diskussion 


\section{ARTIKELANGABEN}

Aundesrat taher Desing

Bundesrat 2011 Mini-Review

Buddeberg e 2008a Quantitativ

Schaufelber 2013 Mini-Review

Tandjung et -2013b Recherche Scholat

Djalali et al. 2013 Mini-Review Scholar

8adertscher 2011a Quantitativ Scholar

Badertscher $2011 \mathrm{~b}$ Quantitativ Schola

Buddeberg e 2007a Qualit. Inhaltsanalyse Scholar

Buddeberge 2006 Quantitativ Scholar

Buddeberg e 2008b Quantitativ Scholar

Buddeberg e $200 \mathrm{7b}$. Qualit. Inhaltsanalyse Scholar

Buddeberge 2011 Quantitativ u. Qualit. Scholar

Burch et al. 2013 Quantitativ u. Qualit. Scholar

Burghardt et 2012 Qualit. Intialtsanalyse AAe

Halter et al. 2005 Quantitativ Scholar

Hänsgen 2007 Quantitativ Scholar

Hasler 2006 Deskriptive Statistik FMH

Hasler, Reich 2009 Deskriptive Statistik FMH

Reichert 2010 Deskriptive Statistik FMH

Rosemann 2012 Report

Scholar

* Künzi, Strub 2012 Deskriptive Statistix. FMH

Marty et al. 2006 Deskriptive Statistik HA

Marty et al. 2007 Quantitativ
EXTAINSISCHE FAXTOREN ("Realitot")

Arbeits- Arbeits- Okonomie, Weiter- Ausbildung. Arbeits- Arbeits- Fionraphie, Psychische Publikat. Struktur inhalte 5oziałzat bilduns Med.Soz. struktur inhaite Privatieben Soz Selekt. Mothe

Bericht
Journal

journal

Journal

Joumal

Journal

Journat

Journal

Journal

Journal

Journal

Journgl

Journal

Journal

Journal

Openkecen

Journal

Journal

Guathten

Gutachten

Gutachten

Journal

Gutactiten

Journat

Journal

\begin{tabular}{|l|}
\hline 0 \\
\hline \\
\hline 0 \\
\hline 0 \\
\hline 0 \\
\hline 000 \\
\hline 000 \\
\hline 00 \\
\hline 0 \\
\hline
\end{tabular}

\begin{tabular}{|c|c|c|c|c|c|c|}
\hline \multirow[t]{4}{*}{$\overline{1}$} & 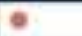 & 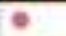 & & & - & \\
\hline & & 0 & $\bullet$ & & • & . \\
\hline & 000 & & & & & \\
\hline & ๑. & ec & & & + & \\
\hline$\bullet$ & & & & & 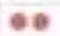 & \\
\hline \multicolumn{7}{|l|}{60} \\
\hline \multicolumn{7}{|l|}{ eee } \\
\hline \multicolumn{7}{|l|}{$\bullet 0$} \\
\hline \multicolumn{7}{|l|}{000} \\
\hline \multirow[t]{2}{*}{$\bullet$} & . & & & & & \\
\hline & . & & & 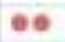 & 0 & ee \\
\hline .. & . & & & $\bullet$ & 0 & -0 \\
\hline$\bullet$ & $\bullet \bullet$ & & & & & \\
\hline \multirow[t]{4}{*}{$\bullet$} & & e & e. & $\bullet$ & & \\
\hline & & 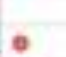 & $\bullet$ & & * & \\
\hline & - & $\bullet$ & & & & \\
\hline & & & & & $\bullet$ & \\
\hline & & & & & & \\
\hline & & & & & & \\
\hline & & & & & & \\
\hline \multicolumn{7}{|l|}{000} \\
\hline & & & & & & \\
\hline & & & & & & \\
\hline & & & & & & \\
\hline
\end{tabular}

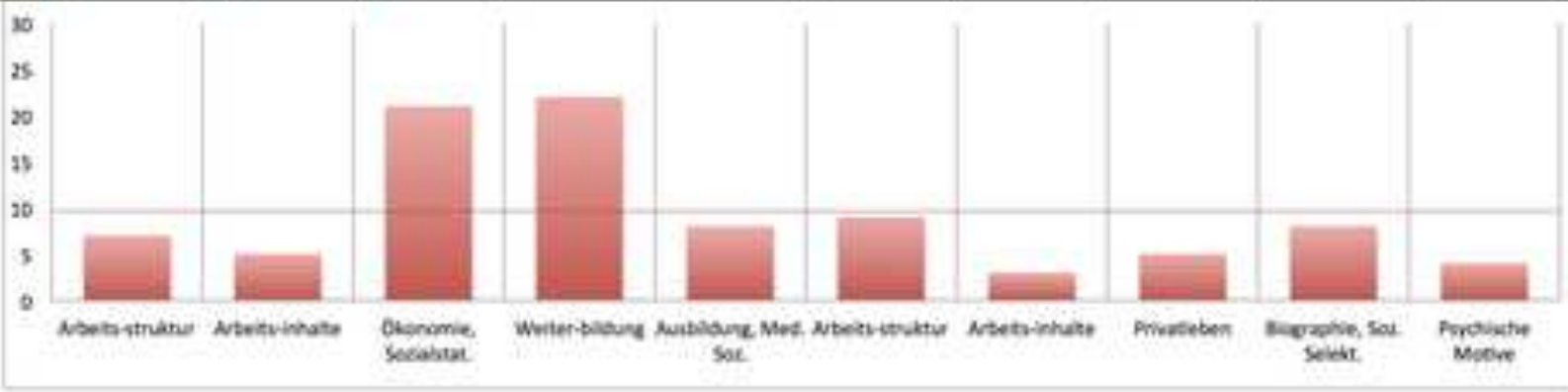

Legende: der Kategorie sind gewidmet...

- mind, 1 Abschnitt (mir Öbersthitit) 
Appendix 3: Ursachen des Hausärzte (HA)-Mangels nach Kategorien, Frankreich.

In Standardschrift HA-Mangel-Ursachen, in Kursivschrift wichtige Zusatzinformationen. Hervorhebungen fett. AA meint Assistenzärzte ungeachtet der Fachrichtung, MedG-AA bezeichnet Assistenzärzte in der Fachrichtung Médecine générale.

\begin{tabular}{|c|c|}
\hline Kategorie & $r w$ \\
\hline Arbeitsinhalte & $\begin{array}{l}\text { - Grundsätzlich überzeugt die HA-Medizin mit ihren Inhalten: das breite Krankheitsspektrum und die Arzt-Patienten-Beziehung sind } \\
\text { die zwei mit Abstand am häufigsten genannten Motivationsfaktoren für die HA-Medizin-Wahl [1-3]. } \\
\text { - Dennoch stellt Navarro [4] unter den Medizinstudenten auch eine abschreckend Wirkung des breiten Krankheitsspektrums fest: } \\
\text { nämlich die Angst vor der Verantwortung alle Details kennen zu müssen in einer immer spezialisierteren Medizin. Zudem stehe es } \\
\text { im Widerspruch mit dem Kompetenzbedürfnis. } \\
\text { - Navarro[4] berichtet von der Bewertung der HA-Medizin-Inhalte als „Bobologie", besonders durch Studenten ohne absolviertes HA- } \\
\text { Medizin-Praktikum. Bloy [5] entwindet den Studenten eine nähere Beschreibung von „Bobologie“: wenig schlimme, banale Krankheiten } \\
\text { als Arbeitsgut. } \\
\text { - Bloy [5] berichtet von MedG-AA nach absolvierter HA-Medizin-Praxisassistenz, denen das Verkleidetsein der medizinischen Leiden in } \\
\text {-BlaBla“ missfällt, d.h. die emotionaleren Patienten-Bekundungen im hausärztlichen Setting. } \\
\text { Decorde [6] stellt fest: Administrative Aufgaben sind häufig genannter Bremsfaktor der Niederlassung als HA. }\end{array}$ \\
\hline $\begin{array}{l}\text { Arbeits- } \\
\text { strukturen }\end{array}$ & $\begin{array}{l}\text { - Zahlreiche landesweite Arbeiten [3, 7-10] untersuchen ausführlich die Wünsche der MedG-AA bezüglich der Arbeitsstrukturen. } \\
\text { Das neue Arbeitsideal ist für 60-70\% der AA die (interdisziplinäre) Gruppenpraxis, die sozialen \& fachlichen Austausch, flexiblere } \\
\text { Arbeitszeiten und Teilen der Investitionen ermöglicht. Die Autonomie der HA-Tätigkeit übt eine grosse Anziehung aus. } \\
\text { - Andere Autoren [6, 11-13] untersuchen die realen Arbeitsstrukturen der bereits niedergelassenen HA. Junge HA arbeiten zu 67\% in } \\
\text { Gruppenpraxen (zu 13\% in interdisziplinären), bei den alten HA ist der Anteil tiefer. Die durchschnittliche Arbeitszeit liegt bei 57h/Wo, } \\
\text { in Gruppenpraxen durchschnittlich 2-4h/Wo. weniger. } \\
\text { - Regierung und Soziologen [3, 9] stellen fest, dass sich die realen Arbeitsbedingungen noch zu wenig mit den Wünschen decken. Zum } \\
\text { Beispiel wollen 70\% der MedG-AA durchschnittlich } 12 \mathrm{~h} \text { weniger arbeiten als die alten HA[3]. } \\
\text { - Bismuth [1] und Bloy [14] konstatieren, dass berufliche Isolation und schwer kontrollierbare Arbeitszeiten auf 50\% der jungen HA, } \\
\text { die Vertretungen machen, abschreckend wirken. Sie zählen zu den } 3 \text { häufigsten Demotivatoren der Niederlassung. } \\
\text { - Navarro [4] erhebt eine Angst vor einem „verdorbenem Leben durch den Notfalldienst“. } \\
\text { - Gemäss Rouger [2] betrachten die AA an der HA-Medizin als unattraktiv die im Vergleich zu den Spezialisten schlechtere } \\
\text { - } \text { Bebensqualität. } \\
\text { Bloy [5] berichtet von MedG-AA, denen die Abhängigkeit vom Patienten im liberalen Setting missfällt. Die Patienten sind dort mehr als } \\
\text { im Krankenhaus freiwillige Kunden deren Unzufriedenheit ökonomische Folgen haben kann. } \\
\text { Demotivierend wirkt auch der Verlust v. beruflicher Autonomie bei zunehmender Ressourcenkontrolle durch Versicherer und Politik } \\
\text { [15]. }\end{array}$ \\
\hline $\begin{array}{l}\text { Ökonomie/ } \\
\text { Sozialstatus }\end{array}$ & $\begin{array}{l}\text { - Bellamy [16] stellt fest, dass die HA-Löhne tief sind im Vergleich zu anderen ambulanten Disziplinen. Der Durchschnitt aller } \\
\text { ambulanten Löhne liegt } 2008 \text { bei 92'500 Euro, Maximum verdienen Radiologen (220'000 } € \text { ), Minimum (60'000 €) Dermatologen und }\end{array}$ \\
\hline
\end{tabular}


Psychiater, die HA kommen auf 70'000 Euro (Platz 12/15).

- Rouger [2] und Navarro [4] erheben, dass der im Vergleich zu Spezialisten schlechtere HA-Lohn die HA-Medizin-Wahl unter den jungen AA unattraktiv macht. Gemäss Schweyer [3] beklagen MedG-AA, dass Freunde aus anderen Berufen mit kürzerer Studienzeit mehr verdienen.

- Decorde [6] berichtet, dass Praxis-bedingte Investitionen und wiederkehrende Kosten von den MedG-AA zu den fünf häufigsten Demotivatoren der HA-Niederlassung gezählt werden.

Bloy [5] identifiziert eine Subgruppe von MedG-AA, die durch HA-Medizin-Arbeitsinhalte angezogen, aber durch die Praxis-bedingten Investitionen abgeschreckt werden. Letztere erzwingen eine bestimmte Stundenzahl/Woche über eine bestimmte Anzahl von Jahren.

- Schweyer [3] stellt fest, dass der Staat durch Schaffung lukrativer Krankenhausstellen für HA-Medizin-Diplomierte (in Geriatrie, Notaufnahme, Psychiatrie) diese aus dem ambulanten Bereich abwirbt. Es locken gute Löhne und geregelte Arbeitszeiten.

- Die Weiterbildung in FR ist grundsätzlich national einheitlich strukturiert und setzt sich aus 6-monatigen klinischen Rotationen und ca. 200 Stunden Theorie zusammen [17].

- Dennoch stellt Angot [18] fest, dass nur 49\% der MedG-AA mit ihrer Weiterbildung zufrieden sind. $57 \%$ wollen mehr ambulante Weiterbildungszeit (Praxisassistenz), 58\% befürworten hierfür eine Verlängerung auf 4 Jahre. So wären innen zudem Rotationen in Gynäkologie und Pädiatrie möglich (bisher entweder-oder).

- Den Wunsch nach mehr Praxisassistenz bestätigt Franco [19], eine nationale Umfrage [8] findet derweil einen gravierenden Mangel an Lehrärzten: 2011 konnten nur 30\% der Internes die 2. Praxisassistenz machen.

- Dabei wird berichtet, dass die Praxisassistenz einen neuralgischen Punkt in der Entscheidung hin zur Grundversorgungstätigkeit darstellt. Strukturierte und EBM-geleitete Lehrärzte brechen Vorurteile und inspirieren zur Tätigkeit als HA, „laissez-faire“-HA hingegen schrecken die AA von der HA-Praxis ab [5, 19].

- Gemäss Franco [19] bemängeln die MedG-AA, dass die theoretischen Weiterbildungsblöcke kaum psychosoziale Fertigkeiten schulen, obwohl sie ein wichtiges Werkzeug des HA darstellen.

- Spiess [17] zeigt auf, dass die Schulung in praktischen Aspekten der Praxisführung sehr unterschiedlich ist. 70\% der MedG-AA kriegen Unterricht bezgl. Durchführung einer Niederlassung und Rechnungslegung, die wenigsten hingegen werden über die Organisation und Möglichkeiten der Fortbildung orientiert.

Ausbildung

- Die Ausbildung wird von 74\% der Medizinstudenten als Einflussfaktor ihrer Fachrichtungs-Wahl angegeben [20].

- Dabei berichten viele Autoren [4, 19, 21] von einer Quasi-Absenz der HA-Medizin im Studium. 80\% der Studenten geben an, HAMedizin-Realität nicht zu kennen, 70\% geben an, dass HA-Medizin zu wenig unterrichtet wird. Von 11'500 Studenten machten 2007 nur 395 ein HA-Medizin-Praktikum.

- Winckler [22] konstatiert, dass eine segmentierte, spezialisierte und Technik-lastige Ausbildung nur spezialisierte, Technik-orientierte Ärzte hervorbringen kann. Die die HA-Medizin auszeichnenden Inhalte fehlten.

- Studentenorganisationen bemängeln, dass keine Ausbildungsinhalte dem Führen einer Praxis gewidmet sind. Diese Herausforderung werde so zum abschreckendem Faktor [9].

- Hardy [23] erklärt die Bedeutung von Wertesystemen im französischen Medizinstudium. Mehr als alle Vorläufer erfordert das 2004 eingeführte ECN-System das Entscheiden anhand von "Proxys": Die Fachrichtungswahl kann nicht mehr auf der Basis realer Erfahrungen während der Assistenzzeit getroffen werden, sondern muss bereits am Studienende anhand von Repräsentationen 


\begin{tabular}{|c|c|}
\hline & 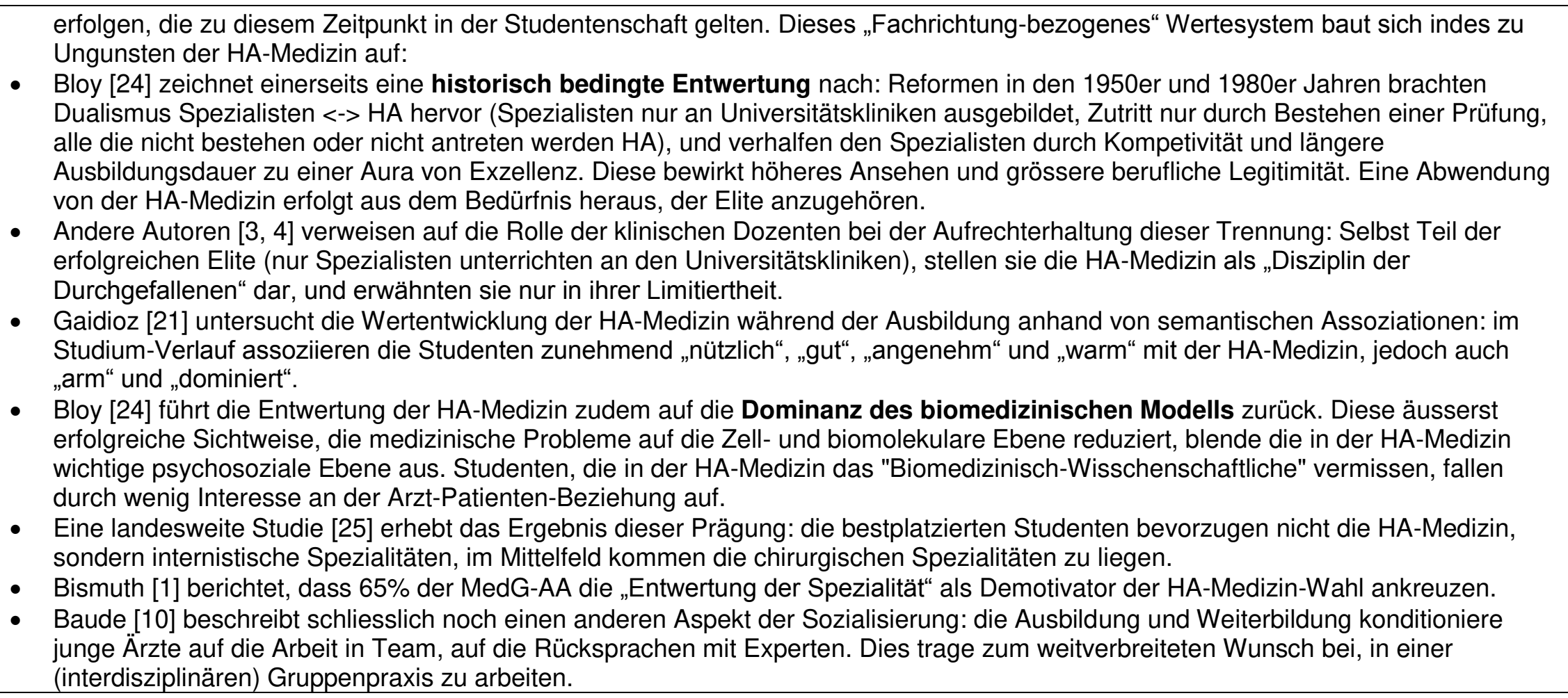 \\
\hline Privatleben & $\begin{array}{l}\text { - Schweyer [3] setzt die Berufstätigkeit der Frau und die daraus resultierende neue Paar- und Familiensoziologie in Zusammenhang mit } \\
\text { der HA-Medizin-Krise. Es erfolge eine Neudefinition des Berufsmodells aus dem Privatleben heraus: Frauen arbeiten auch } \rightarrow \text { altes } \\
\text { HA-Modell „Mann ist 150\% für Patienten da, Frau kümmert sich um Haushalt und Kinder“ funktioniert nicht mehr. } \\
\text { - Schweyer [3] stellt weitergehend fest, dass Männer im Zuge der neuen Geschlechterrollen mehr über ihre Zeiteinteilung nachdenken. } \\
\text { Die Freizeit gewinne gegenüber dem Beruf an Bedeutung als identitätsstiftendes Element, was ein zeitintensives unternehmerisches } \\
\text { HA-Modell noch weiter schwäche. } \\
\text { - Schliesslich ist ein neues „Primat des Familienlebens“ unter den MedG-AA feststellbar (78\% geben Familienleben gegenüber Beruf } \\
\text { den Vorzug). Das Modell des aufopferungsvollen HA erscheint erneut wenig attraktiv [3]. }\end{array}$ \\
\hline $\begin{array}{l}\text { Biografie/ } \\
\text { Soz.Selektion }\end{array}$ & $\begin{array}{l}\text { - Gallois [15] sieht in der Feminisierung der Medizin eine Teilursache des aktuellen HA-Mangels. Die damit verbundenen } \\
\text { Vorstellungen der Frauen bezüglich Gleichgewicht Arbeit <-> Privatleben seien inkompatibel mit dem Arbeitsmodell der alten HA. } \\
\text { - Gaucher [20] und Schweyer [3] stellen Zusammenhang fest zwischen sozialer Selektion durch Studium (Studenten zu 59\% } \\
\text { Intellektuellenkinder, zu 1-2\% Bauernkinder) und der Fachrichtungwahl. Besonders Ärztekinder wenden sich von HA-Medizin ab und } \\
\text { suchten „Wege der Exzellenz“ in den internistischen Spezialitäten; im Gegensatz dazu wenden sich Mittelklasse-Studenten häufiger }\end{array}$ \\
\hline
\end{tabular}


der HA-Medizin zu, die für sie einen sozialen Aufstieg bedeute.

Psychische

Motive

- Bloy [14] sieht die Niederlassung als HA im Widerspruch zur „generationellen Norm der europäischen Jugend“, die sich durch Ausprobieren von Möglichkeiten, Aufschieben von Engagement und Vermeiden des Definitiven auszeichne. Demgegenüber böten Krankenhaus-Stellen und Vertretungen diese Unverbindlichkeit.

- Schweyer [3] berichtet ebenfalls von einer Angst vor vorzeitiger Verwurzelung unter den MedG-AA durch die HA-Praxistätigkeit.

- Bloy [14] konstatiert ein wachsendes Freiheitsbedürfnis unter den Medizinabsolventen nach Abschluss des französischen Studiums mit seinen „Zahlreichen Zwängen und blinder Selektion“ [blind gegenüber inhaltlichen Wünschen]. Diese Gegenreaktion verstärke zusätzlich die generationelle Norm des sich-nicht-festlegen-Wollens. 
Appendix 4: Ursachen des Hausärzte (HA)-Mangels nach Kategorien, Schweiz.

In Standardschrift HA-Mangel-Ursachen, in Kursivschrift wichtige Zusatzinformationen. Hervorhebungen fett. Wo nicht näher eingegrenzt steht AA für Assistenzärzte ungeachtet der Fachrichtung.

\section{Einschlägige Arbeiten}

Arbeitsinhalte

- Grundsätzlich überzeugt die HA-Medizin mit ihren Inhaltsrealitäten. Das breite Krankheitsspektrum und die Arzt-Patient Beziehung (individuell, ganzheitlich, kontinuierlich) sind unter Studenten und AA die stärksten Argumente für die HA-Medizin [26-29].

- Dennoch stellt Buddeberg [30] fest, dass gerade das breite Krankheitsspektrum auf einige Jung-Ärzte unattraktiv wirkt: Der Wissensfortschritt macht es schwierig, alles zu wissen, die Spezialisierung erscheint als Weg, kompetent zu bleiben.

- Als häufige Argumente gegen die HA-Medizin werden ausserdem genannt: Weiterweisung der spannenden Fälle, steigende Gefahr, Fehler zu machen, und das Fehlen von herausfordernden Interventionen [26].

Arbeitsstrukturen
- Mehrere Arbeiten [28, 31, 32] befragen Medizinstudenten und junge Ärzte nach den gewünschten künftigen Arbeitsbedingungen. Das ideale Arbeitssetting stellt die Gruppenpraxis dar (für 84\% der Studienabgänger), die interdisziplinäre Gruppenpraxis als auch die „Gemeinde-vermietete“ Gruppenpraxis erscheinen als attraktive Modifikationen, besonders auf dem Land. Die Flexibilität der Arbeitsgestaltung und unternehmerische Freiheit als HA übt eine grosse Anziehungskraft aus [27, 29].

- Ein Bericht des Bundesrats [33] befindet, dass die aktuelle ambulante Versorgungsstruktur den Bedürfnissen der neuen Generation nicht entspricht. Studienabgänger geben als häufigstes Argument gegen die HA-Medizin-Wahl ihre Arbeitsbedingungen an [26].

- Entsprechend bezeichnen Medizinstudenten als wichtigste Massnahme zur Attraktivitätssteigerung der HA-Medizin die Anderung der Arbeitsbedingungen: Arbeitszeit, Gruppenpraxis, Lohn [31].

- Buddeberg [29] befragt praktizierende HA nach negativen Arbeitsrealitäten. Genannt werden die Angst vor Rekursen im Kampf mit den Krankenversicherungen und die unbefriedigende Organisation und Entschädigung des Notfalldienstes.

- Eine Trendwende zeigt sich bei den Arbeitsstrukturen unter neu niedergelassenen HA: 30\% arbeiten in Einzelpraxen, $70 \%$ in $2 e r$ oder Gruppenpraxen. Frauen sind häufiger in Angestelltenverhältnis und Gruppenpraxen, mit ø um 2 Halbtage kürzeren Arbeitszeiten [34]. 
Ökonomie /

Sozialstatus
- Es besteht eine Einkommensschere zwischen niedergelassenen Allgemeinärzten und Spezialisten [35-37]. Diese geht seit der TARMED-Einführung 2004 nicht weiter auf, persistiert jedoch unverändert. Noch 2011 stellt ein Bundesratsbericht fest, dass das TARMED die HA gegenüber den Spezialisten benachteilige [33].

- Künzi und Strub [38] berechnen das AHV-pflichtige Median-Einkommen der niedergelassenen HA (2009: 197 '500 CHF). 12 Spezialisten-Fächer verdienen signifikant mehr (höchstes Median-Einkommen: 374'350, Gastroenterologie), 14 Spezialisierungen verdienen ähnlich, 3 Fachrichtungen verdienen signifikant weniger (tiefstes Medianeinkommen: 107'400, Kinder- und Jugendpsychiatrie).

- $\quad$ Burch [39] und Burghardt [26] stellen fest, dass „geringes Einkommen“ als einer der drei meistgenannten Gründe gegen die HAMedizin-Wahl angeführt wird. Dies ist schon unter den Medizinstudenten des 1. Studienjahres der Fall.

- Die Kombination aus niedrigerem Realeinkommen und gleichzeitig bestehendem unternehmerischem Risiko der Praxistätigkeit wirkt auf die potenziellen jungen HA abschreckend [28].

- Buddeberg [29] stellt eine berufsübergreifende Tendenz zur Spezialisierung fest. Diese spreche „Allroundern“, wie z.B. HA, weniger Prestige zu. Tatsächlich berichtet Burch [39], dass 30\% der befragten Medizinstudenten „fehlende soziale Anerkennung“ als Hindernis der HA-Medizin-Wahl betrachten.

- Buddeberg [40] verortet in der Tatsache, dass die Schweiz kein starkes Gatekeeper-Modell hat (wie z.B. England, Niederlande), einen Signifikanzverlust der HA-Medizin. Die unsichere Entwicklung der Stellung der HAM wirke zudem auf junge Ärzte abschreckend [28, 41].

- $\quad$ Tandjung [34] und Buddeberg [28] zeigen die grosse Bedeutung die Weiterbildungszeit für den Berufsentscheid auf. Je nach Studie entschieden $54 \%$ bis $75 \%$ der jungen HA erst während ihrer Assistenzzeit HA zu werden. Die Autoren erklären dies damit, dass erst in der Weiterbildung der Wert des breiten Patientenspektrums und der ganzheitlichen Langzeitbetreuung erkannt wird.

- Dabei zeigt sich, dass die Weiterbildung zum HA in der Schweiz im Vergleich zu anderen Spezialitäten und zu anderen Ländern bisher wenig attraktiv und weniger zielführend ist: Sie ist selbst zu organisieren, beinhaltet wenig HA-Medizin-spezifische Inhalte und bedingt während der Praxisassistenz häufig Lohneinbussen von bis zu 25\% [28, 34, 40, 42]. Zudem fordert sie oft geografische oder zeitliche Opfer, weil kurze klinische Rotationen (z.B. in Pädiatrie), wie sie für den HA lehrreich wären, an Zentrumsspitälern schwierig zu ergattern sind.

- Badertscher [43] untersucht die Weiterbildung näher auf ihre HA-Medizin-Spezifität. Die Mehrheit der künftigen HA in internistischen oder chirurgischen Kliniken gibt an, keine spezifischen Lernziele besprochen zu haben mit ihren Vorgesetzten. Nur bei $48 \%$ finden HA-Medizin-spezifische Weiterbildungen statt und bei nur $31 \%$ ist eine HA-Praxisassistenz möglich.

- Tandjung [44] stellt fest, dass zwar HA-Praxisassistenzprogramme zunehmend quantitativ ausgebaut werden, HA-Medizin-spezifische Curricula („Gesamtpakete“) mit im Voraus organisierten klinischen Rotationen jedoch weiterhin selten sind Somit fehlten nach wie vor Weiterbildungsstellen für HA-Medizin, die organisatorische und finanzielle Planungssicherheit bieten.

- Buddeberg [30] untersucht die Transition Weiterbildung -> Niederlassung. 8 J. nach Studienabschluss sind nur 38\% der HA-Anwärter in der Praxis, der Rest arbeitet als Assistenz- oder Kaderarzt. Als Motivatoren der Niederlassung gelten die Praxisassistenz und eine Mentorbeziehung zu einem HA, als Hemmer die hohen Kosten der Praxisübernahme und die Schwierigkeit, eine Hypothek zu kriegen. 


\begin{tabular}{|c|c|}
\hline Ausbildung & $\begin{array}{l}\text { - Badertscher[43] und Buddeberg[45] stellen fest, dass Erfahrungen während des Studiums wichtig sind für den späteren Wunsch, } \\
\text { HA zu werden. 30\% der angehenden HA geben an, durch Kontakt mit der HA-Medizin während dem Studium motiviert worden zu sein. } \\
\text { - Halter [32] und Isler [46] zeigen, dass sich der Prozentsatz der Studenten mit HA-Wunsch während des Einzeltutorats verdoppelt bis } \\
\text { verdreifacht, jedoch verschwindet dieser Effekt bis Studienende wieder und zeigt keine Auswirkung auf die Facharzttitelvergabe. } \\
\text { - Buddeberg [40] stellt fest, dass im Medizinstudium die HA-Medizin zeitlich untervertreten ist, die Vorlesungen wirkten weniger } \\
\text { professionell. Der Bundesrat [33] kommt zum gleichen Schluss und empfiehlt die Stärkung von HA-Medizin-Inhalten in Studium. } \\
\text { - Studienabsolventen bemängeln das Fehlen von HA-Medizin-relevanten betriebswirtschaftlichen und gesundheitsökonomischen } \\
\text { Inhalten im Studium [28]. }\end{array}$ \\
\hline $\begin{array}{l}\text { Medizinische } \\
\text { Sozialisation }\end{array}$ & $\begin{array}{l}\text { - In medizinischer Fachwelt und Öffentlichkeit liegt der Fokus zu sehr auf der spezialisierten Spitzenmedizin, während die Bedeutung der } \\
\text { HA-Medizin unreflektiert bleibt [31]. } \\
\text { - Zudem erlebten Studenten eine Geringschätzung der HA-Medizin durch Spitalärzte und Dozenten [31]. Die HA-Medizin-Anwärter } \\
\text { bekunden einen Mangel an positiven Vorbildern, die den abschätzigen Bemerkungen der Spitalärzte entgegenwirken würden [40]. } \\
\text { - Schaufelberger [47] berichtet, dass im HA-Praktikum eine Patienten-zentrierte medizinische Sozialisierung erfolge. }\end{array}$ \\
\hline Privatleben & $\begin{array}{l}\text { - Buddeberg }[30,45,48] \text { untersucht die Vorstellungen und Realitäten bezgl. Privatleben von künftigen und jungen HA. Gegenüber } \\
\text { früheren HA-Generationen verschob sich das Gleichgewicht Arbeit <-> Privatleben deutlich zu Gunsten der Familie. Gegenüber } \\
\text { Spezialisten sind HA häufiger verheiratet, haben häufiger Kinder und wählen häufiger das Berufsmodell „Vater \& Mutter Teilzeit“ } \\
\text { anstelle eines zeitintensiven karriereorientierten Modells. }\end{array}$ \\
\hline $\begin{array}{l}\text { Biografie/ } \\
\text { Soz. Selektion }\end{array}$ & $\begin{array}{l}\text { - Tandjung [34] beschreibt den „neuen HA“ als wahrscheinlich weiblich, in Gruppenpraxis Teilzeit arbeitend und angestellt. } \\
\text { - Buddeberg [48] findet keine statistisch signifikanten Unterschiede zwischen jungen HA vs. Spezialisten bezüglich } \\
\text { soziodemographischem Hintergrund. Väter von jungen Spezialisten sind in der betrachteten Stichprobe zwar doppelt so häufig Ärzte } \\
\text { wie Väter von jungen HA, jedoch ist der Unterschied aufgrund insgesamt kleiner Fallzahl nicht signifikant. } \\
\text { - Medizinstudenten mit HA-Eltern(teil) beurteilen die Arbeitsstrukturen der HA-Medizin negativer als solche ohne HA-Eltern, dafür haben } \\
\text { sie mehr positive Nennungen in der Kategorie "Arbeitsinhalte“. Letztlich wählen sie aber nicht häufiger/seltener HA-Medizin als } \\
\text { Berufsziel [26]. }\end{array}$ \\
\hline $\begin{array}{l}\text { Psychische } \\
\text { Motive }\end{array}$ & $\begin{array}{l}\text { - Buddeberg [45, 48] untersucht die psychische Ausstattung und Motivationsstruktur von künftigen HA. Karriere und Prestige sind ihnen } \\
\text { weniger wichtig als künftigen Spezialisten, dafür umso mehr Familie und Freizeit. Die subjektive Beurteilung ihres Karriere-Erfolges fällt } \\
\text { niedriger aus als bei den Spezialisten. } \\
\text { - Buddeberg [28] stellt zwei psychische Hindernisse der HA-Niederlassung unter Assistenzärzten fest: } \\
\text { die Angst vor definitiver Festlegung und die Angst vor der Verantwortung als HA. }\end{array}$ \\
\hline
\end{tabular}




\section{Bibliographie}

1. Bismuth $M$. Pourquoi les internes choisissent-ils la médecine générale: La médecine générale revient dans les premiers choix. Médecine. 2013:136-41.

2. Rouger A-S. La médecine générale: un choix par défaut aux épreuves classantes nationales? Étude multicentrique sur la concrétisation des souhaits des étudiants aux ECN. Thèse pour le doctorat en médecine. Rouen: Université de Rouen; 2013.

3. Schweyer F-X. Souhaits et aspirations des médecins qui envisagent l'exercise de la médecine générale. In: ONDPS, editor. Rapport 2006-2007, Tome 1: La médecine générale. Paris: Observatoire National de la Démographie des Professions de Santé (ONDPS); 2008. p. 89-135.

4. Navarro L, Dubois J-P. Désintérêt des étudiants de 6 e année pour la médecine générale: une étude auprès de 15 volontaires lyonnais. La revue du practicien médecine générale. 2007;21:466-7.

5. Bloy G. La transmission des savoirs professionnels en médecine générale : le cas du stage chez le praticien. Revue Française des Affaires Sociales. 2005:101-25.

6. Decorde A. Déterminants à l'installation en médecine générale: analyse croisée des médecins généralistes remplaçants et jeunes installés du sécteur nord alpin. Thèse pour l'obtention du doctorat en médecine. Grenoble: Université Joseph Fourier; 2011.

7. Augros S. Les conditions de travail souhaitées par les futurs médecins généralistes: étude nationale déscriptive réalisée auprès des internes de médecine générale du 2 juillet 2010 au 1 Janvier 2011. Thèse pour l'obtention du doctorat en Médecine. Grenoble: Université Joseph Fourier; 2012.

8. ISNAR-IMG. Enquête nationale sur les souhaits d'exercice des internes de médecine générale: résultats. Lyon: Inter syndicale nationale autonome représentative des internes de médecine générale, 2011.

9. ORS-Aquitaine. Motivations et freins à l'installation des médecins généralistes libéraux: synthèse de la littérature. Bordeaux: Observatoire régional de la santé d'Aquitaine, 2011.

10. Baude N, Flacher A, Bosson J-L, Marchand O. Soins primaires: crise et dynamique d'avenir: Les attentes des internes de troisième cycle de médecine générale. Médecine. 2008;4:135-40.

11. Baudier F, Bourgueil Y, Evrard I, Gautier A, Le Fur P, Mousquès J. La dynamique de regroupement des médecins généralistes libéraux de 1998 à 2009. Questions d'économie de la santé 2010:2-6.

12. Jakoubovitch S, Bournot M-C, Cercier E, Tuffreau F. Les emplois du temps des médecins généralistes. Série Études et résultats n 797. Paris: Direction de la recherche, des études, de l'évaluation et des statistiques, Ministère des Affaires sociales et de la Santé; 2012.

13. Aulagnier $M$, Obadia $Y$, Paraponaris $A$, et.al. L'exercice de la médecine générale libérale: Premiers résultats d'un panel dans cinq régions françaises. Série Études et résultats $n^{\circ} 610$. Paris: Direction de la recherche, des études, de l'évaluation et des statistiques, Ministère des Affaires sociales et de la Santé; 2007.

14. Bloy G. Comment peut-on devenir généraliste aujourd'hui? Le renouvellement des médecins généralistes vu à travers une cohorte de jeunes diplômés. Revue française des affaires sociales. 2011:9-28.

15. Gallois P, Vallée J-P, Le Noc Y. Médecine générale en crise: faits et questions. Médecine. 2006;2:223-8.

16. Bellamy V. Les revenus libéraux des médecins en 2007 et 2008. Série Études et résultats $n^{\circ} 735$. Paris: Direction de la recherche, des études, de l'évaluation et des statistiques, Ministère des Affaires sociales et de la Santé; 2010.

17. Spiess J. État des lieux des enseignements des spécificités de la pratique de la médecine générale en libéral dans les différentes facultés de médecine de France 
métropolitaine. Thèse pour le doctorat en médecine. Rouen: Université de Rouen 2013.

18. Angot $O$. Faut-il réformer l'internat de médecine générale? La revue du practicien médecine générale. 2007;21:633-4.

19. Franco Rodrigues A. Représentations et conceptions de la médecine générale: regards croisés entre internes et médecins généralistes. Thèse pour le diplôme d'état de docteur en médecine. Paris: Université Pierre et Marie Curie (Paris 6); 2012.

20. Gaucher S, Thabut D. [Medical specialty choice: What impact of teaching? Results of a survey of two medical schools.]. Presse Médicale. 2013;42:e89-e95.

21. Gaidioz C, Ruhlmann S. Pourquoi la spécialité médecine générale est-elle mal classée aux choix des épreuves classantes nationales? Thèse pour obtenir le grade de Docteur en Médecine. Lyon: Université Claude Bernard LYON I; 2008.

22. Winckler M. La crise de la médecine générale. Les Tribunes de la santé. 2009:67-74.

23. Hardy-Dubernet A-C. À propos d'une minute décisive: le choix d'une spécialité médicale. Journal de gestion et d'économie médicales. 2009;27:174-90.

24. Bloy G. Une médecine générale durablement dépréciée? Enquête sur une évidence. In: ONDPS, editor. Rapport 2006-2007, Tome 1: La médecine générale. Paris:

Observatoire National de la Démographie des Professions de Santé (ONDPS); 2008. p. 137-73.

25. Lefèvre J, Karila L, Kernéis S, Fiessinger J-N, Rouprêt M. Désintérêt des futurs médecins pour la médecine générale: Résultats d'une enquête nationale sur les choix de spécialisation auprès de 1870 externes français. Presse Médicale. 2010:e58e65.

26. Burghardt L, Dreisbach M, Shahla H, Businger A. Swiss Student's Arguments in Favour and against a Career in Primary Care. Primary Health Care. 2012;2.

27. Hasler L, Stamm M, Buddeberg-Fischer B. Zukünftige Hausärztinnen und Hausärzte: Gründe für die Berufswahl und berufliche Kernkompetenzen. Praxis. 2008;97:127785.

28. Buddeberg-Fischer B, Stamm M, Buddeberg C, Klaghofer R. Möglichkeiten zur Attraktivitätssteigerung der Hausarztmedizin aus der Sicht junger Ärztinnen und Ärzte. Gesundheitswesen. 2008a;70:123-8.

29. Buddeberg-Fischer B, Stamm M, Marty F. Attraktivitätssteigerung der Hausarztmedizin: Ansichten und Vorschläge von praktizierenden Hausärzten. Primary Care. 2007a;7:639-41.

30. Buddeberg-Fischer B, Klaghofer R, Stamm M. Family Physicians in Switzerland: Transition From Residency to Family Practice. Family Medicine. 2011;43:29-36.

31. Tschudi P, Bally K, Zeller A. [Who wants to be a GP? A survey medical students and junior doctors]. Praxis. 2013;102:335-9.

32. Halter U, Tschudi P, Bally K, Isler R. Berufsziel von Medizinstudierenden. Primary Care. 2005;5:468-72.

33. Bundesrat. Strategie gegen Ärztemangel und zur Förderung der Hausarztmedizin: Bericht des Bundesrates in Erfüllung der Motion 08.3608 von Nationalrätin Jacqueline Fehr „Strategie gegen Ärztemangel und zur Förderung der Hausarztmedizin“ vom 2. Oktober 2008. Bern 2011.

34. Tandjung R, Senn O, Marty F, Kraussa L, Rosemann T, Badertscher N. Career after successful medical board examination in general practice: a cross-sectional survey. Swiss Medical Weekly. 2013a;143.

35. Hasler N. Einkommensverhältnisse der freien Ärzteschaft der Schweiz in den Jahren 2001 und 2002. Schweizerische Ärztezeitung. 2006;87:87-93.

36. Hasler N, Reichert M. Einkommensverhältnisse der freien Ärzteschaft der Schweiz in den Jahren 2005 (neu) und 2004 (Re-Evaluation). Schweizerische Ärztezeitung. 2009;90:409-19.

37. Reichert M. Einkommensverhältnisse der freien Ärzteschaft der Schweiz in den Jahren 2006 (neu) und 2005 (Re-Evaluation). Schweizerische Ärztezeitung. 2010;91:479-87. 
38. Künzi K, Strub S. Einkommen der Ärzteschaft in freier Praxis: Auswertung der Medisuisse-Daten 2009 Schweizerische Ärztezeitung. 2012;93:1-5.

39. Burch M-A, Cochard B, Corpataux N, Passaplan C, Rayroux C. Pénurie en médecins de premier recours dans le canton de Fribourg: Regard croisé des médecins généralistes établis dans le canton et des étudiants en médecine de Fribourg. Revue Médicale Suisse. 2013:1328-9.

40. Buddeberg-Fischer B, Stamm M, Marty F. Family medicine in Switzerland: training experiences in medical school and residency. Family Medicine. 2007b;39:651-5.

41. Marty F, König U, Sutter J, Betschart M. Motivation junger Ärzte, Grundversorger zu werden. Primary Care. 2007;7:50-3.

42. Djalali S, Frei A, Rosemann T, Tandjung R.

Weiterbildung zum Hausarzt - ein europäischer Vergleich. Praxis. 2013;102:327-33.

43. Badertscher N, Schöni M, Berger L, Zoller M, Rosemann T. [Vocational training of future GPs in Swiss hospitals: the view of assistant physicians]. Praxis.

2011a;100:349-55.

44. Tandjung R, Garaventa-Tadres D, Rosemann T, Djalali S. Spezifische Weiterbildungsangebote für Hausarztmedizin in der Schweiz: Bestandsaufnahme verschiedener kantonaler Programme. Praxis. 2013b;102:843-9.

45. Buddeberg-Fischer B, Stamm M, Buddeberg C, Klaghofer R. The new generation of family physicians: career motivation, life goals and work-life balance. Swiss Medical Weekly 2008b;138:305-12.

46. Isler R, Romerio S, Halter U, et.al. Das Einzeltutorat in Basel: eine zehnjährige Erfolgsgeschichte. Primary Care. 2009;9:74-7.

47. Schaufelberger M. Attraktivitätssteigerung der Grundversorgung im Medizinstudium. Praxis. 2013;102:341-4.

48. Buddeberg-Fischer B, Klaghofer R, Stamm M, et.al. Primary Care in Switzerland: no longer attractive for young physicians? Swiss Medical Weekly. 2006a;136:416-24. 